hep-ph/0404020

KUNS-1906

November 8, 2018

\title{
Horizontal symmetry in Higgs sector of GUT with $U(1)_{A}$ symmetry
}

\author{
Nobuhiro Maekawa ${ }^{\mathrm{a}}$ and Toshifumi Yamashita ${ }^{\mathrm{b}}$ \\ Department of Physics, Kyoto University, \\ Kyoto 606-8502, Japan
}

\begin{abstract}
In a series of papers, we pointed out that an anomalous $U(1)_{A}$ gauge symmetry naturally solves various problems in grand unified theories (GUTs) and that a horizontal gauge symmetry, $S U(2)_{H}$ or $S U(3)_{H}$, not only realizes the unification of three generation quarks and leptons in fewer multiplets but also solves the supersymmetric flavor problem. In this paper, we examine the possibility that the Higgs sectors of the GUT symmetry and of the horizontal symmetry are unified, that is, there are some Higgs fields whose vacuum expectation values (VEVs) break both the GUT gauge symmetry and the horizontal symmetry at the same time. Although the scale of the VEVs become too large to suppress the flavor changing neutral current processes sufficiently, the unification is possible. In addition, for the $S U(3)_{H}$ models, the $S U(3)_{H}$ gauge anomaly is cancelled in the unified models without introducing additional fields in contrast with the previous models in which the Higgs sectors are not unified.
\end{abstract}

\footnotetext{
${ }^{a}$ E-mail: maekawa@gauge.scphys.kyoto-u.ac.jp

${ }^{b}$ E-mail: yamasita@gauge.scphys.kyoto-u.ac.jp
} 


\section{Introduction}

We have several reasons for introducing a horizontal symmetry $G_{H}$. One of them is to understand the origin of the flavor violation in Yukawa couplings of quarks and leptons. Actually many studies have been done along this direction[1]-8].

The second reason is to unify quarks and leptons in fewer multiplets, though it is strongly related with the first motivation. Usual Grand unified theory (GUT) has two kinds of unification. The first unification is to unify gauge interactions, and the second is to unify quarks and leptons. However, usual GUT with $S U(5), S O(10)$ or $E_{6}$ gauge group can unify only one generation quarks and leptons. In order to unify all the quarks and leptons into a single multiplet, a larger gauge group such as $S O(12+2 n), E_{7}$, or $E_{8}$ is required, though these unified group cannot realize chiral matter in a 4-dimensional theory. However, actually it is possible in higher dimensional field theories, and in that cases, a horizontal symmetry can appear in the effective 4-dimensional field theories.

The third reason is to solve the flavor problem in supersymmetric theories [9]. A nonabelian flavor (horizontal) symmetry can potentially solve the problem. If the first two generation fields become a doublet under the flavor symmetry, $\Psi_{a}(a=1,2)$, it is obvious that, unless the flavor symmetry is broken, the sfermion masses of the first two generation fields become universal, which is important in solving the SUSY flavor problem. Of course, in order to obtain realistic hierarchical structure of Yukawa couplings, the flavor symmetry must be broken, for example, by the vacuum expectation value (VEV) $\left\langle F_{a}\right\rangle$. Then, generically the universal sfermion masses are lifted by the breaking. Various models in which the breaking effects can be controlled have been considered in the literature 10, 11, 12. However, in GUT models with bi-large neutrino mixings, which have been reported by recent experiments [13, 14, the universality of the first two generation sfermion masses is not sufficient to solve the SUSY flavor problem, because the large mixings and the $\mathcal{O}(1)$ discrepancy between the sfermion masses of the third generation fields and those of the first two generation fields lead to too large flavor changing neutral current (FCNC) processes, for example, $\epsilon_{K}$ in $K$ meson, $\mu \rightarrow e \gamma, \tau \rightarrow \mu \gamma$, etc. One of the authors pointed out that $E_{6}$ unification can naturally solve this problem in Refs. [15]. The essential point is that in the $E_{6}$ unification all three light modes of $\overline{\mathbf{5}}$ fields come from the first two generation fields

$\Psi_{a}(27)[3]$, because it is naturally expected that the $\overline{\mathbf{5}}$ fields from the third generation field $\Psi_{3}$ become superheavy owing to its large Yukawa couplings. Therefore, all the three light modes of $\overline{\mathbf{5}}$ have universal sfermion masses, which are important in solving the SUSY flavor problem.

In a series of papers [4, 5, 6] [16]-[19, we proposed an attractive GUT scenario with an anomalous $U(1)_{A}$ gauge symmetry [20], whose anomaly is cancelled by the Green-Schwarz mechanism[21]. One of the biggest differences between our works and previous works is to introduce generic interactions even for higher dimensional interactions. Therefore, once we fix the symmetry of the theory, we can define the theory except $\mathcal{O}(1)$ coefficients. It is interesting that under this natural assumption, the doublet-triplet (DT) splitting is realized with sufficiently stable nucleon, and realistic quark and lepton mass matrices including the bi-large neutrino mixings are obtained. Moreover, the non-abelian horizontal gauge symmetry can be naturally introduced in the GUT scenario with an anomalous $U(1)_{A}$ gauge symmetry to solve the SUSY flavor problem, especially in the $E_{6}$ unification. 
In Refs. 15], the Higgs fields to break the horizontal gauge symmetry $G_{H}=S U(2)_{H}$ or $S U(3)_{H}$ are different from the Higgs fields to break the GUT gauge group $S O(10)$ or $E_{6}$. However, in principle, these Higgs sector can be unified, namely, a non-vanishing VEV of a Higgs field with non-trivial representation for both gauge groups can break both gauge groups. If it is realized, the number of the Higgs fields can be reduced. In this paper, we examine this possibility.

After this introduction, we give a brief review of the horizontal symmetry that is introduced to suppress the FCNC processes in section 2. And in section 3, we examine the possibility for $S O(10)$ unification, and in section $4, E_{6}$ unification is examined.

\section{Horizontal symmetry for SUSY flavor problem}

In this section, we briefly review an idea that a horizontal symmetry is introduced to solve the SUSY flavor problem, because this is one of the most important motivation to introduce a horizontal symmetry.

For simplicity, we consider a simple model with a global horizontal symmetry $U(2)$, under which the three generations of quarks and leptons, $\Psi_{i}=\left(\Psi_{a}, \Psi_{3}\right)(a=1,2)$, are transformed as $\mathbf{2}+\mathbf{1}$, and the Higgs field $H$ is a singlet. Then only the Yukawa interaction $\Psi_{3} \Psi_{3} H$ is allowed by the horizontal symmetry, which accounts for the large top Yukawa coupling. Suppose that the $U(2)$ horizontal symmetry is broken by the VEVs of a doublet $\left\langle\bar{F}^{a}\right\rangle=\delta_{2}^{a} V$ and of an anti-symmetric tensor $\left\langle A^{a b}\right\rangle=\epsilon^{a b} v\left(\epsilon^{12}=-\epsilon^{21}=1\right)$ as

$$
U(2)_{H} \underset{V}{\longrightarrow} U(1)_{H} \underset{v}{\longrightarrow} \text { nothing. }
$$

The ratios of the VEVs to the cutoff, $\epsilon \equiv V / \Lambda \gg \epsilon^{\prime} \equiv v / \Lambda$, give the following hierarchical structure of the Yukawa couplings:

$$
Y_{u, d, e} \sim\left(\begin{array}{ccc}
0 & \epsilon^{\prime} & 0 \\
\epsilon^{\prime} & \epsilon^{2} & \epsilon \\
0 & \epsilon & 1
\end{array}\right) .
$$

Moreover, the $U(2)_{H}$ symmetric interaction $\int d^{4} \theta \Psi^{\dagger a} \Psi_{a} Z^{\dagger} Z$, where $Z$ has a non-vanishing VEV given by $\langle Z\rangle \sim \theta^{2} \tilde{m}$, leads to approximate universality of the first and second generation sfermion masses:

$$
\tilde{m}_{u, d, e}^{2} \sim \tilde{m}^{2}\left(\begin{array}{ccc}
1 & 0 & 0 \\
0 & 1+\epsilon^{2} & \epsilon \\
0 & \epsilon & O(1)
\end{array}\right)
$$

Here, $\epsilon^{2}$ results from higher dimensional interactions, like $\int d^{4} \theta\left(\Psi_{a} \bar{F}^{a}\right)^{\dagger} \Psi_{b} \bar{F}^{b} Z^{\dagger} Z$, through a non-vanishing $\mathrm{VEV}\langle\bar{F}\rangle$. The important parameters which are constrained by the FCNC processes are defined by

$$
\delta_{x} \equiv V_{x}^{\dagger} \frac{\tilde{m}_{x}^{2}-\tilde{m}^{2}}{\tilde{m}^{2}} V_{x}
$$


where $V_{x}$ is a diagonalizing matrix for fermions $x=q, u_{R}, d_{R}, l, e_{R}[22]$. The constraints are given as, for example,

$$
\begin{aligned}
\sqrt{\left.\mid \operatorname{Im}\left(\delta_{D_{L}}\right)_{12}\left(\delta_{D_{R}}\right)_{12}\right) \mid} & \leq 2 \times 10^{-4}\left(\frac{\tilde{m}_{Q}}{500 \mathrm{GeV}}\right) \\
\left|\operatorname{Im}\left(\delta_{D_{R}}\right)_{12}\right| & \leq 1.5 \times 10^{-3}\left(\frac{\tilde{m}_{Q}}{500 \mathrm{GeV}}\right)
\end{aligned}
$$

at the weak scale from $\epsilon_{K}$ in the $K$ meson mixing, and

$$
\left|\left(\delta_{E_{L}}\right)_{12}\right| \leq 4 \times 10^{-3}\left(\frac{\tilde{m}_{L}}{100 \mathrm{GeV}}\right)^{2}
$$

from the $\mu \rightarrow e \gamma$ process.

Actually, the $U(2)_{H}$ symmetry realizes not only hierarchical Yukawa couplings but also approximately universal sfermion masses of the first two generation fields. These mass matrices lead to the relations

$$
\frac{\tilde{m}_{2}^{2}-\tilde{m}_{1}^{2}}{\tilde{m}^{2}} \sim \frac{m_{F 2}}{m_{F 3}},
$$

where $m_{F i}$ and $\tilde{m}_{i}$ are the masses of the $i$-th generation fermions and the $i$-th generation sfermions, respectively. Unfortunately, these predictions of this simple model imply a problematic contribution to the $\epsilon_{K}$ parameter in the $K$ meson mixing and the $\mu \rightarrow e \gamma$ process. Moreover, it is obvious that the hierarchical Yukawa couplings predicted by this simple model are similar for the up-quark sector, the down-quark sector, and the charged-lepton sector. This is inconsistent with the experimental results. Moreover, in the neutrino sector, it seems to be difficult to obtain the large neutrino mixing angles that have been measured in some recent experiments [13, 14].

One of the most natural solutions for the above problems is to introduce $E_{6}$ unification. One of the points is that the Cabibbo-Kobayashi-Maskawa (CKM) mixings are obtained from the mixings of the diagonalizing matrix of $\mathbf{1 0}$ fields of $S U(5)$ that includes the doublet quark $Q$ and the Maki-Nakagawa-Sakata (MNS) mixings are obtained from the mixings of the diagonalizing matrix of $\overline{\mathbf{5}}$ fields of $S U(5)$ that includes the doublet lepton $L$. The fundamental representation 27 of $E_{6}$ is divided as

$$
\mathbf{2 7} \rightarrow \underbrace{\left[1 \mathbf{1 0}_{1}+\overline{\mathbf{5}}_{-3}+\mathbf{1}_{5}\right]}_{16_{1}}+\underbrace{\left[\overline{\mathbf{5}}_{2}+\mathbf{5}_{-2}\right]}_{\mathbf{1 0 _ { - 2 }}}+\underbrace{\left[\mathbf{1}_{0}\right]}_{\mathbf{1}_{4}}
$$

under $E_{6} \supset S O(10) \times U(1)_{V^{\prime}} \supset S U(5) \times U(1)_{V} \times U(1)_{V^{\prime}}$. Here, the representations of $S O(10) \times U(1)_{V^{\prime}}$ and $S U(5) \times U(1)_{V}$ are explicitly denoted. Therefore, if we introduce three $27_{i}(i=1,2,3)$ for the three generation quarks and leptons, only three of the six $\overline{\mathbf{5}}$ fields represent quarks and leptons in our world and the other $\overline{\mathbf{5}}$ s become superheavy. One of the most important assumptions is that the main light modes of $\overline{\mathbf{5}}$ fields come from the first two generation fields $27_{1}$ and $27_{2}[3$. The assumption that $\overline{\mathbf{5}}$ fields from the third generation field $\mathbf{2 7} 3$ has larger couplings, that is, larger masses is natural because the third generation field $\mathbf{2 7}_{3}$ must have larger Yukawa coupling to realize the large top 
Table 1: Typical values of anomalous $U(1)_{A}$ charges. The symbols \pm denote a $Z_{2}$ parity.

\begin{tabular}{|c|c|c|}
\hline & non-vanishing VEV & vanishing VEV \\
\hline $\mathbf{4 5}$ & $A(a=-1,-)$ & $A^{\prime}\left(a^{\prime}=3,-\right)$ \\
$\mathbf{1 6}$ & $C(c=-3,+)$ & $C^{\prime}\left(c^{\prime}=2,-\right)$ \\
$\overline{\mathbf{1 6}}$ & $\bar{C}(\bar{c}=0,+)$ & $\bar{C}^{\prime}\left(\bar{c}^{\prime}=5,-\right)$ \\
$\mathbf{1 0}$ & $H(h=-3,+)$ & $H^{\prime}\left(h^{\prime}=4,-\right)$ \\
$\mathbf{1}$ & $\Theta(\theta=-1,+), Z(z=-2,-), \bar{Z}(\bar{z}=-2,-)$ & $S(s=3,+)$ \\
\hline
\end{tabular}

quark mass. Then, actually, the $\overline{\mathbf{5}}$ fields can have milder hierarchical Yukawa couplings than the 10 fields, and therefore, the MNS mixings become larger than the CKM mixings. When we introduce a horizontal symmetry $S U(2)_{H}$, all the three generation $\overline{\mathbf{5}}$ fields come from a single field $\mathbf{2 7 _ { a }}$, which results in universal sfermion masses for $\overline{\mathbf{5}}$ fields. Note that the $E_{6}$ symmetry is sufficient but not necessary for the above structure. The sufficient and necessary symmetry is $S U(2)_{E}$ that rotates two $\overline{\mathbf{5}}_{\mathrm{s}}$ and two $\mathbf{1}$ s in $\mathbf{2 7}$ representation as doublets.

\section{$3 S O(10) \times S U(2)_{H}$}

In this section, we consider a model with an $S O(10) \times S U(2)_{H} \times U(1)_{A}$ gauge symmetry. This model does not solve the SUSY flavor problem sufficiently, but it is shown that in the Higgs sector, the horizontal symmetry can be naturally introduced.

\subsection{Doublet-triplet splitting in $S O(10)$}

In this subsection, we review a mechanism that realizes the DT splitting naturally in the $S O(10)$ unified scenario without a non-abelian horizontal gauge symmetry. 4]

The content of the Higgs sector with the $S O(10) \times U(1)_{A}$ gauge symmetry is given in Table 1, where the symbols "+" and " - " denote $Z_{2}$ parity quantum numbers.

One of the most important features of the GUT scenario with the anomalous $U(1)_{A}$ symmetry is that VEVs are determined by anomalous $U(1)_{A}$ charges as

$$
\left\langle O_{i}\right\rangle \sim\left\{\begin{array}{cc}
\lambda^{-o_{i}} & o_{i} \leq 0 \\
0 & o_{i}>0
\end{array},\right.
$$

where the $O_{i}$ are GUT gauge singlet operators with charges $o_{i}$, and $\lambda \equiv\langle\Theta\rangle / \Lambda \ll 1$. Here the Froggatt-Nielsen (FN) field $\Theta$ has an anomalous $U(1)_{A}$ charge of -1 [23]. Throughout this paper, we denote all superfields and chiral operators by uppercase letters and their anomalous $U(1)_{A}$ charges by the corresponding lowercase letters. When convenient, we use units in which $\Lambda=1$. Such a vacuum structure is naturally obtained if we introduce generic interactions even for higher-dimensional operators and if the $F$-flatness conditions determine the scale of the VEVs 4, 5]. Since all the positively charged singlet operators have vanishing VEVs, the SUSY zero (holomorphic zero) mechanism acts effectively. Namely, the negatively charged interactions are not allowed by the symmetry. 
Therefore the superpotential for determination of the VEVs can be written as

$$
\begin{aligned}
W & =W_{H^{\prime}}+W_{A^{\prime}}+W_{S}+W_{C^{\prime}}+W_{\bar{C}^{\prime}}, \\
W_{A^{\prime}} & =\lambda^{a^{\prime}+a} A^{\prime} A+\lambda^{a^{\prime}+3 a}\left(\left(A^{\prime} A\right)_{1}\left(A^{2}\right)_{1}+\left(A^{\prime} A\right)_{\mathbf{5 4}}\left(A^{2}\right)_{\mathbf{5 4}}\right), \\
W_{S} & =\lambda^{s+c+\bar{c}} S\left((\bar{C} C)+\lambda^{-(c+\bar{c})}+\lambda^{-(c+\bar{c})+2 a} A^{2}\right), \\
W_{C^{\prime}} & =\bar{C}\left(\lambda^{\bar{c}^{\prime}+c+a} A+\lambda^{\bar{c}^{\prime}+c+\bar{z}} \bar{Z}\right) C^{\prime}, \\
W_{\bar{C}^{\prime}} & =\bar{C}^{\prime}\left(\lambda^{\bar{c}^{\prime}+c+a} A+\lambda^{\bar{c}^{\prime}+c+z} Z\right) C, \\
W_{H^{\prime}} & =\lambda^{h+a+h^{\prime}} H^{\prime} A H .
\end{aligned}
$$

Here $W_{X}$ denotes the terms linear in the field $X$ that has positive anomalous $U(1)_{A}$ charge and we omit $\mathcal{O}(1)$ coefficients. Note that terms including two fields with positive charge, like $\lambda^{2 h^{\prime}} H^{\prime} H^{\prime}$, give contributions to the mass terms but not to the VEVs. In Eq. (11), the suffices $\mathbf{1}$ and $\mathbf{5 4}$ indicate the representations of the composite operators under the $S O(10)$ gauge symmetry. In the above equations, for simplicity, we ignore terms like $\mathbf{1 6} \mathbf{6}^{\mathbf{4}}$, $\overline{16}^{4}, 10 \cdot 16^{2}, 10 \cdot \overline{16}^{2}$ and $1 \cdot 10^{2}$, even if they are allowed by the symmetry. This is because these interactions do not play a significant role in our argument, because they do not include products of only the neutral components under the standard gauge group. It is easy to include these terms in our analysis.

The $F$-flatness condition of $A^{\prime}$ have a solution in which $\langle A\rangle=i \tau_{2} \times \operatorname{diag}(v, v, v, 0,0)$, which breaks $S O(10)$ into $S U(3)_{C} \times S U(2)_{L} \times S U(2)_{R} \times U(1)_{B-L}$. It is interesting that the scale $v$ is determined by the anomalous $U(1)_{A}$ charge as $v \sim \lambda^{-a}$. This DimopoulosWilczek (DW) form of the VEV plays an important role in solving the DT splitting problem [24].

The $F$-flatness condition of $S$ determines the scale of the VEV $\langle\bar{C} C\rangle$ as $\langle\bar{C} C\rangle \sim$ $\lambda^{-(c+\bar{c})}$, and the $D$-flatness condition requires $|\langle C\rangle|=|\langle\bar{C}\rangle| \sim \lambda^{-(c+\bar{c}) / 2}$. The scale of the VEV is again determined by only the charges of $C$ and $\bar{C}$.

The $F$-flatness conditions of $C^{\prime}$ and $\bar{C}^{\prime}$ realize the alignment of the VEVs $\langle C\rangle$ and $\langle\bar{C}\rangle$ (Barr-Raby mechanism) 25]. The $F$-flatness conditions $F_{C^{\prime}}=F_{\bar{C}^{\prime}}=0$ give $\left(\lambda^{a-z} A+\right.$ $Z) C=\bar{C}\left(\lambda^{a-\bar{z}} A+\bar{Z}\right)=0$. Recall that the VEV of $A$ is proportional to the $B-L$ generator $Q_{B-L}$ as $\langle A\rangle=\frac{3}{2} v Q_{B-L}$. Also $C, \mathbf{1 6}$, is decomposed into $(\mathbf{3}, \mathbf{2}, \mathbf{1})_{1 / 3},(\overline{\mathbf{3}}, \mathbf{1}, \mathbf{2})_{-1 / 3}$, $(\mathbf{1}, \mathbf{2}, \mathbf{1})_{-1}$ and $(\mathbf{1}, \mathbf{1}, \mathbf{2})_{1}$ under $S U(3)_{C} \times S U(2)_{L} \times S U(2)_{R} \times U(1)_{B-L}$. Since $\langle\bar{C} C\rangle \neq 0$, not all components in the spinor $C$ vanish. Then $Z$ is fixed as $Z \sim-\frac{3}{2} \lambda v Q_{B-L}^{0}$, where $Q_{B-L}^{0}$ is the $B-L$ charge of the component field that has the non-vanishing VEV in $C$. It is interesting that no other component fields can have non-vanishing VEVs, because of the $F$-flatness conditions. If the $(\mathbf{1}, \mathbf{1}, \mathbf{2})_{1}$ field obtains a non-zero VEV (and therefore $\left.\langle Z\rangle \sim-\frac{3}{2} \lambda v\right)$, then the gauge group $S U(3)_{C} \times S U(2)_{L} \times S U(2)_{R} \times U(1)_{B-L}$ is broken to the standard gauge group. Once the direction of the VEV $\langle C\rangle$ is determined, the VEV $\langle\bar{C}\rangle$ must have the same direction, because of the $D$-flatness condition. Therefore, $\langle\bar{Z}\rangle \sim-\frac{3}{2} \lambda v$. Thus, all VEVs have now been fixed.

Finally, the $F$-flatness condition of $H^{\prime}$ is examined. The $F$-flatness condition $F_{H^{\prime}}=0$ leads to the vanishing VEV of the triplet Higgs, $\left\langle H_{T}\right\rangle=0$.

Here, we examine the mass spectrum of $\mathbf{5}$ and $\overline{\mathbf{5}}$ of $S U(5)$, to see how the DT splitting is realized. Considering the additional terms $H^{\prime} H^{\prime}, \bar{C} A \bar{C}^{\prime} H, \bar{C} \bar{C}^{\prime} H^{\prime}, C C^{\prime} H^{\prime}, H^{\prime} \bar{C} \bar{C} A$ and $\bar{C}^{\prime} C^{\prime}$ we write the mass matrices $M_{I}$, which are for the representations $I=D^{c}\left(H_{T}\right), L\left(H_{D}\right)$ 
Table 2: Typical values of anomalous $U(1)_{A}$ charges.

\begin{tabular}{|c|c|}
\hline$S O(10) \times S U(2)_{H}$ & $A(a=-1 / 2), \quad A^{\prime}\left(a^{\prime}=3 / 2\right)$ \\
\hline$(\mathbf{4 5}, \mathbf{1})$ & $B(b=-7 / 2)$ \\
$(\mathbf{1}, \mathbf{3})$ & $C(c=-1)$ \\
$(\mathbf{1 6}, \mathbf{2})$ & $\bar{C}(\bar{c}=5 / 2)$ \\
$(\overline{\mathbf{1 6}}, \overline{\mathbf{2}})$ & $H(h=-9 / 2), \quad H^{\prime}\left(h^{\prime}=5\right)$ \\
$(\mathbf{1 0 , 1})$ & $Z(z=-3 / 2), \quad S(s=2), \quad S^{\prime}\left(s^{\prime}=13 / 2\right)$ \\
$(\mathbf{1}, \mathbf{1})$ & $F(f=-3 / 2), \quad F^{\prime}\left(f^{\prime}=2\right)$ \\
$(\mathbf{1}, \mathbf{2})$ & \\
\hline
\end{tabular}

and their conjugates:

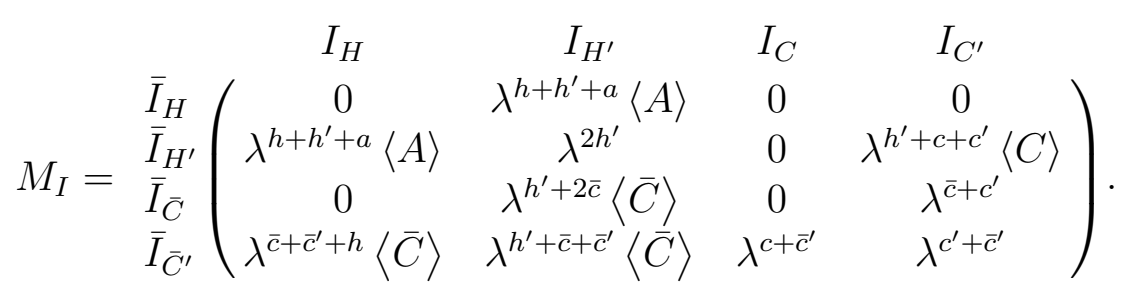

In this matrix, $\langle A\rangle$ vanishes only for doublet Higgs, so only one pair of doublet Higgs becomes massless. Massless modes are

$$
\begin{aligned}
H_{D} & =\overline{\mathbf{5}}_{H}+\lambda^{\bar{c}-c+h}\langle\bar{C}\rangle \overline{\mathbf{5}}_{C}, \\
\bar{H}_{D} & =\mathbf{5}_{H} .
\end{aligned}
$$

Namely the DT splitting is realized. The effective colored Higgs mass is estimated as $\left(\lambda^{h+h^{\prime}}\right)^{2} / \lambda^{2 h^{\prime}}=\lambda^{2 h}$, which is larger than the cutoff scale, because $h<0$. Therefore the proton decay via dimension 5 operators is naturally suppressed.

Straightforward calculation shows that all the non-singlet fields become massive except one pair of doublet Higgs discussed above. Surprisingly, though the mass spectrum does not respect the $S U(5)$ GUT symmetry, the gauge coupling unification can be naturally realized. This is because the general discussions for the natural gauge coupling unification in Refs. [18] can be applied. This gauge coupling unification requires that the cutoff scale $\Lambda$ must be around the usual GUT scale $\Lambda_{G} \sim 2 \times 10^{16} \mathrm{GeV}$ and the unification scale becomes $\lambda^{-a} \Lambda$, which makes proton decay via dimension 6 operators interesting.

\subsection{Doublet-triplet splitting in $S O(10) \times S U(2)_{H}$}

The content of Higgs sector is listed in Table 2. The essential part of the superpotential to determine the vacuum structure is written as

$$
\begin{aligned}
W= & A^{\prime} A+A^{\prime} A^{3}+\bar{C}(A+Z) C+H^{\prime} A H+S(1+\bar{C} B C) \\
& +F^{\prime}(\bar{C} C) F+S^{\prime}(1+F B F+\bar{C} B C+\bar{C} F C F) .
\end{aligned}
$$


Here we omit the $\mathcal{O}(1)$ coefficients as well as the power factor $\lambda^{x}$ where $x$ is the total charge of the corresponding term. The half integer charges play the same role as $Z_{2}$ parity in the previous $S O(10)$ model. The last term in Eq.(19) and fields $F$ and $F^{\prime}$ are required to obtain realistic quark and lepton mass matrices, but not required to realize the DT splitting. Besides the last term, the structure is essentially the same as in the previous models. The difference appears in the terms $\bar{C}(A+Z) C$ and $S(1+\bar{C} B C)$. Since $c+\bar{c}>0$, the VEV $\langle\bar{C} C\rangle$ must have vanishing VEV. On the other hand, the $F$-flatness condition of $S$ leads to the non-vanishing $\mathrm{VEV}\langle\bar{C} B C\rangle \sim \lambda^{-(\bar{c}+b+c)}$. Therefore $\bar{C}, B$ and $C$ must have non-vanishing VEVs. If we take

$$
\begin{aligned}
& \langle C\rangle=\left(\begin{array}{c}
0 \\
V_{C}
\end{array}\right), \quad\langle\bar{C}\rangle=\left(\begin{array}{ll}
V_{\bar{C}} & 0
\end{array}\right), \\
& \langle B\rangle=\left(\begin{array}{cc}
0 & V_{B} \\
0 & 0
\end{array}\right)
\end{aligned}
$$

with $\left|V_{C}\right|=\left|V_{\bar{C}}\right|=\left|V_{B}\right| \sim \lambda^{-\frac{1}{3}(c+\bar{c}+b)}$, it is easily checked that not only the above conditions from $F$-flatness conditions but also the $D$-flatness conditions for $S O(10)$ and $S U(2)$ gauge interactions are satisfied.

The $F$-flatness condition of $\bar{C}$

$$
(A+Z) C=\left(Q_{B-L} \lambda^{a}+Z \lambda^{z}\right) C=0
$$

realizes alignment (Barr-Raby mechanism). As in the previous subsection, The VEV of $Z$ is fixed such that $Z \sim-\frac{3}{2} \lambda v Q_{B-L}^{0}$, where $Q_{B-L}^{0}$ is the $B-L$ charge of the component of $C$ that has non-vanishing VEV. Note that once we fix the $\mathrm{VEV}$ of $Z$, the $F$-flatness condition of $\bar{C}$ automatically realizes the same alignment for the $\mathrm{VEV}$ of $C$, which is consistent with the $D$-flatness condition of $S O(10)$. Namely, an additional sliding singlet $\bar{Z}$ does not required. This interesting feature is realized by unifying the Higgs sectors for breaking $S U(2)_{H}$ and $S O(10)$.

Considering the additional terms $H^{\prime} H^{\prime}, \bar{C}^{2} A H, C^{2} \bar{C}^{2}, H^{\prime} C C$ and $\bar{C}^{2}(1+B A) H^{\prime}$, we write the mass matrices of $\mathbf{5}$ and $\overline{\mathbf{5}}$ of $S U(5)$, which are for the representations $I=$ $D^{c}\left(H_{T}\right), L\left(H_{D}\right)$ and their conjugates:

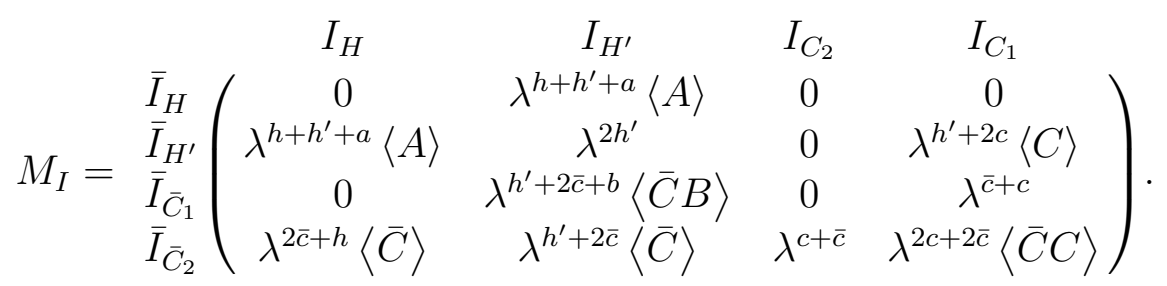

In this matrix, $\langle A\rangle$ vanishes only for doublet Higgs, so only one pair of doublet Higgs

$$
\begin{aligned}
H_{D} & =\overline{\mathbf{5}}_{C_{2}}+\frac{\lambda^{c+\bar{c}}}{\lambda^{2 \bar{c}+h}\langle\bar{C}\rangle} \overline{\mathbf{5}}_{H}, \\
\bar{H}_{D} & =\mathbf{5}_{H}
\end{aligned}
$$

becomes massless. This mixing in the doublet Higgs plays an important role in obtaining quark and lepton mass matrices. 
Table 3: The typical values of anomalous $U(1)_{A}$ charges for quarks and leptons are listed. The odd quarter integer charges play the same role as odd $R$-parity.

\begin{tabular}{|c|c|c|c|}
\hline & $\Psi$ & $\Psi_{3}$ & $T$ \\
\hline$S O(10)$ & $\mathbf{1 6}$ & $\mathbf{1 6}$ & $\mathbf{1 0}$ \\
$S U(2)_{H}$ & $\mathbf{2}$ & $\mathbf{1}$ & $\mathbf{1}$ \\
$U(1)_{A}$ & $\psi=29 / 4$ & $\psi_{3}=9 / 4$ & $t=15 / 4$ \\
\hline
\end{tabular}

The $F$-flatness condition of $F^{\prime}$ leads to

$$
\langle F\rangle=\left(\begin{array}{c}
0 \\
V_{F}
\end{array}\right), \quad V_{F} \sim \lambda^{-f-\frac{1}{3} b+\frac{1}{6}(c+\bar{c})},
$$

where the non-vanishing scale $V_{F}$ is determined by the $F$-flatness condition of $S^{\prime}$ that leads to $\langle F B F\rangle \sim \lambda^{-(2 f+b)}$. Again, an alignment happens by the $F$-flatness condition. This non-vanishing VEV is important in obtaining realistic mass matrices.

When the VEV relation (9) holds, as discussed in Ref. [18, the effective charges $\tilde{x}$ of fields $X$ are defined as

$$
\tilde{x}=x+c_{F} \Delta f+c_{V} \Delta c
$$

where $c_{F}$ and $c_{V}$ are $U(1)_{F} \subset S U(2)_{F}$ and $U(1)_{V}$ charges of $X$ which are normalized by the charges of the component with non-vanishing $\operatorname{VEVs}\langle F\rangle$ and $\langle C\rangle$, respectively. Note that the GUT and flavor singlet operators $O=\prod Y_{i}$ have the effective charges $\tilde{o}_{i}=o_{i}$ because $\sum c_{F}\left(Y_{i}\right)=\sum c_{V}\left(Y_{i}\right)=0 . \Delta f$ and $\Delta c$ are determined by the relations

$$
\langle X\rangle \sim \lambda^{-\tilde{x}}
$$

where $X=\mathbf{1}_{\bar{C}}, \mathbf{1}_{C}, \mathbf{1}_{F}, \cdots$, as

$$
\Delta f=\frac{1}{6}(c+\bar{c})-\frac{1}{3} b, \quad \Delta c=-\frac{1}{2}(c-\bar{c}) .
$$

It is easily checked that the effective charges defined in the above determine all the scales of non-vanishing VEVs as $\langle X\rangle \sim \lambda^{-\tilde{x}}$ and masses of superheavy fields of $Y_{1}$ and $Y_{2}$ as $\lambda^{\tilde{y}_{1}+\tilde{y}_{2}}$. Note that all the terms that reproduce the mass term contribute $\mathcal{O}\left(\lambda^{\tilde{y}_{1}+\tilde{y}_{2}}\right)$ to the mass of $Y_{1}$ and $Y_{1}$. For example, $\lambda^{y_{1}+y_{2}+\sum x_{i}} Y_{1} Y_{2} \prod X_{i}=\lambda^{\tilde{y}_{1}+\tilde{y}_{2}+\sum \tilde{x}_{i}} Y_{1} Y_{2} \prod X_{i}$ give $\lambda^{\tilde{y}_{1}+\tilde{y}_{2}} Y_{1} Y_{2}$ by developing the $\operatorname{VEVs}\left\langle X_{i}\right\rangle \sim \lambda^{-\tilde{x}_{i}}$. It is obvious that even for higer dimensional interactions, the coefficients are determined by the simple sums of the effective charges. Thus, in the followings, we often write down only some representatives of interactions.

\subsection{Quark and lepton sector}

It is not so difficult to obtain realistic quark and lepton mass matrices, using this simple Higgs sector. For example, let us introduce the matter sector as in Table 3, Here the rational charges play the same role as $R$-parity. 
The $\mathbf{1 0}$ field is divided into $\mathbf{5}+\overline{\mathbf{5}}$ of $S U(5)$, thus one pair of $\mathbf{5}+\overline{\mathbf{5}}$ becomes massive. First of all, we examine which modes become massless. The mass matrix $M_{I}$ of $\mathbf{5}$ and $\overline{\mathbf{5}}$ are determined by the effective charges

$$
\begin{aligned}
\tilde{\psi}_{1}(\overline{\mathbf{5}}) & \equiv \psi-\frac{3}{5} \Delta c+\Delta f=\frac{29}{4}+\frac{11}{30} \\
\tilde{\psi}_{2}(\overline{\mathbf{5}}) & \equiv \psi-\frac{3}{5} \Delta c-\Delta f=\frac{29}{4}-\frac{37}{15} \\
\tilde{\psi}_{3}(\overline{\mathbf{5}}) & \equiv \psi_{3}-\frac{3}{5} \Delta c=\frac{9}{4}-\frac{21}{20} \\
\tilde{t}(\overline{\mathbf{5}}) & \equiv t+\frac{2}{5} \Delta c=\frac{15}{4}+\frac{7}{10} \\
\tilde{t}(\mathbf{5}) & \equiv t-\frac{2}{5} \Delta c=\frac{15}{4}-\frac{7}{10}
\end{aligned}
$$

from the interactions

$$
W_{M}=\Psi_{3} T B C F+T \Psi(1+B(A+Z)) C+T^{2}
$$

as

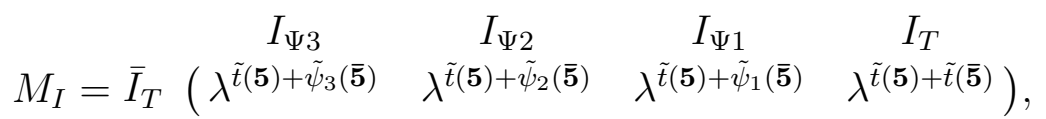

where $I=D^{c}, L$. Here, we do not write down all the interactions that produce the elements of the above matrix, because their contributions have the same power of $\lambda$. Thus, the light modes become

$$
\begin{aligned}
& I_{1} \sim \overline{\mathbf{5}}_{\psi 1}+\lambda^{\tilde{\psi}_{1}(\overline{\mathbf{5}})-\tilde{\psi}_{3}(\overline{\mathbf{5}})} \overline{\mathbf{5}}_{\Psi 3}, \\
& I_{2} \sim \overline{\mathbf{5}}_{\psi 2}+\lambda^{\tilde{\psi}_{2}(\overline{\mathbf{5}})-\tilde{\psi}_{3}(\overline{\mathbf{5}})} \overline{\mathbf{5}}_{\Psi 3}, \\
& I_{3} \sim \overline{\mathbf{5}}_{T}+\lambda^{\tilde{t}(\overline{\mathbf{5}})-\tilde{\psi}_{3}(\overline{\mathbf{5}})} \overline{\mathbf{5}}_{\psi 3}
\end{aligned}
$$

in a certain base of light $\overline{\mathbf{5}}$ fields ${ }^{1}$. Yukawa couplings of quarks and leptons are obtained from the superpotential

$$
\begin{aligned}
W_{Y}= & \Psi_{3}^{2} H+\Psi_{3} \Psi F(A+B) H+\Psi^{2}\left(A Z+F^{2}+A B\right) H \\
& +T \Psi_{3} C F+T \Psi(1+A B) C
\end{aligned}
$$

\footnotetext{
${ }^{1}$ Note that if $F$ has a vanishing VEV, the $\overline{5}_{\Psi 3}$ becomes one of the light modes, that leads to unrealistic quark mass matrices.
} 
as

$$
\begin{aligned}
& Y_{u} \sim \lambda^{\tilde{h} \mathbf{( 5 )}}\left(\begin{array}{lll}
\lambda^{\tilde{\psi}_{1}(\mathbf{1 0})+\tilde{\psi}_{1}(\mathbf{1 0})} & \lambda^{\tilde{\psi}_{1}(\mathbf{1 0})+\tilde{\psi}_{2}(\mathbf{1 0})} & \lambda^{\tilde{\psi}_{1}(\mathbf{1 0})+\tilde{\psi}_{3}(\mathbf{1 0})} \\
\lambda^{\tilde{\psi}_{1}(\mathbf{1 0})+\tilde{\psi}_{2}(\mathbf{1 0})} & \lambda^{\tilde{\psi}_{2}(\mathbf{1 0})+\tilde{\psi}_{2}(\mathbf{1 0})} & \lambda^{\tilde{\psi}_{2}(\mathbf{1 0})+\tilde{\psi}_{3}(\mathbf{1 0})} \\
\lambda^{\tilde{\psi}_{1}(\mathbf{1 0})+\tilde{\psi}_{3}(\mathbf{1 0})} & \lambda^{\tilde{\psi}_{2}(\mathbf{1 0})+\tilde{\psi}_{3}(\mathbf{1 0})} & \lambda^{\tilde{\psi}_{3}(\mathbf{1 0})+\tilde{\psi}_{3}(\mathbf{1 0})}
\end{array}\right),
\end{aligned}
$$

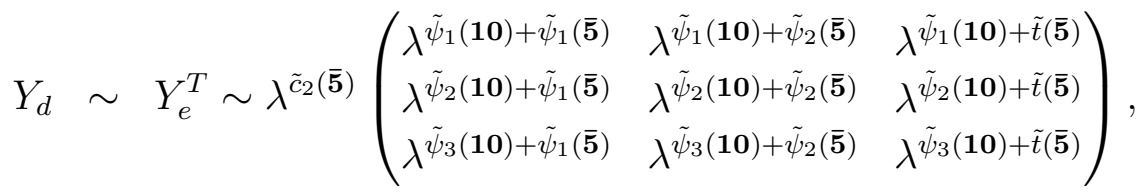

$$
\begin{aligned}
& Y_{\nu_{D}}^{T} \sim \lambda^{\tilde{h}(\mathbf{5})}\left(\begin{array}{lll}
\lambda^{\tilde{\psi}_{1}(\mathbf{1})+\tilde{\psi}_{1}(\overline{\mathbf{5}})} & \lambda^{\tilde{\psi}_{1}(\mathbf{1})+\tilde{\psi}_{2}(\overline{\mathbf{5}})} & \lambda^{\tilde{\psi}_{1}(\mathbf{1})+\tilde{t}(\overline{\mathbf{5}})} \\
\lambda^{\tilde{\psi}_{2}(\mathbf{1})+\tilde{\psi}_{1}(\overline{\mathbf{5}})} & \lambda_{\psi_{2}(\mathbf{1})+\tilde{\psi}_{2}(\overline{\mathbf{5}})} & \lambda^{\tilde{\psi}_{2}(\mathbf{1})+\tilde{t}(\overline{\mathbf{5}})} \\
\lambda^{\tilde{\psi}_{3}(\mathbf{1})+\tilde{\psi}_{1}(\overline{\mathbf{5}})} & \lambda^{\tilde{\psi}_{3}(\mathbf{1})+\tilde{\psi}_{2}(\overline{\mathbf{5}})} & \lambda^{\tilde{\psi}_{3}(\mathbf{1})+\tilde{t}(\overline{\mathbf{5}})}
\end{array}\right) .
\end{aligned}
$$

Note that down-quark and charged lepton Yukawa matrices have additional contribution given through the Higgs mixing in Eq. (24), which is the same order as that from the Higgs $\overline{\mathbf{5}}_{H}$. This is guaranteed by the fact that the matter mixings in Eq. (37) and the Higgs mixing are determined by the difference of the effective charges. For example, $\lambda^{\psi+t+c} \Psi T C$ and $\lambda^{\psi+\psi_{3}+h+f} \Psi F \Psi_{3} H$ give Yukawa interactions $\lambda^{\tilde{\psi}_{1}(\mathbf{1 0})+\tilde{t}(\overline{\mathbf{5}})+\tilde{c}_{2}(\overline{\mathbf{5}})} \mathbf{1 0}_{\Psi_{1}} \overline{\mathbf{5}}_{T} \overline{\mathbf{5}}_{C_{2}}$ and $\lambda^{\tilde{\psi}_{1}(\mathbf{1 0})+\tilde{\psi}_{3}(\overline{\mathbf{5}})+\tilde{h}(\overline{\mathbf{5}})} \mathbf{1 0}_{\Psi_{1}} \overline{\mathbf{5}}_{\Psi_{3}} \overline{\mathbf{5}}_{H}$, respectively, and the ratio of the Yukawa couplings, $\lambda^{\tilde{t}(\overline{\mathbf{5}})+\tilde{c}_{2}(\overline{\mathbf{5}})-\tilde{\psi}_{3}(\overline{\mathbf{5}})-\tilde{h}(\overline{\mathbf{5}})}$ is nothing but the product of the mixing coefficients in Eqs. (24) and (37). As a result, CKM mixings can be obtained as $V_{i j} \sim \lambda^{\left|\tilde{\psi}_{i}(\mathbf{1 0})-\tilde{\psi}_{j}(\mathbf{1 0})\right|}$.

Because The mass matrix of neutrinos is straightforwardly calculated. From the interactions

$$
W=\left(\Psi_{3} \Psi_{3}+\Psi_{3} \Psi(A+B) F+(\Psi(A+B) F)^{2}\right) \bar{C}^{2} B
$$

we obtain the right-handed neutrino mass matrix as

$$
\left(M_{\nu_{R}}\right)_{i j} \sim \lambda^{\tilde{\psi}_{i}(\mathbf{1})+\tilde{\psi}_{j}(\mathbf{1})}
$$

Therefore, light neutrino mass matrix can be estimated from the seesaw relation as

$$
M_{\nu}=Y_{\nu_{D}} M_{\nu_{R}}^{-1} Y_{\nu_{D}}^{T} \frac{\left\langle h_{u}\right\rangle^{2} \eta^{2}}{\Lambda} \sim \lambda^{2 \tilde{h}(\mathbf{5})}\left(\begin{array}{ccc}
\lambda^{2 \tilde{\psi}_{1}(\overline{\mathbf{5}})} & \lambda^{\tilde{\psi}_{1}(\overline{\mathbf{5}})+\tilde{\psi}_{2}(\overline{\mathbf{5}})} & \lambda^{\tilde{\psi}_{1}(\overline{\mathbf{5}})+\tilde{t}(\overline{\mathbf{5}})} \\
\lambda^{\tilde{\psi}_{2}(\overline{\mathbf{5}})+\tilde{\psi}_{1}(\overline{\mathbf{5}})} & \lambda^{2 \tilde{\psi}_{2}(\overline{\mathbf{5}})} & \lambda^{\tilde{\psi}_{2}(\overline{\mathbf{5}})+\tilde{t}(\overline{\mathbf{5}})} \\
\lambda^{\tilde{t}(\overline{\mathbf{5}})+\tilde{\psi}_{1}(\overline{\mathbf{5}})} & \lambda^{\tilde{t}\left(\overline{\mathbf{s}}^{2}\right)+\tilde{\psi}_{2}(\overline{\mathbf{5}})} & \lambda^{2 \tilde{t}(\overline{\mathbf{5}})}
\end{array}\right) \frac{\left\langle h_{u}\right\rangle^{2} \eta^{2}}{\Lambda},
$$

where $\eta$ is a renormalization factor. Therefore, Maki-Nakagawa-Sakata matrix is obtained as

$$
V_{M N S} \sim\left(\begin{array}{ccc}
1 & \lambda^{\tilde{\psi}_{1}(\overline{\mathbf{5}})-\tilde{\psi}_{2}(\overline{\mathbf{5}})} & \lambda^{\tilde{\psi}_{1}(\overline{\mathbf{5}})-\tilde{t}(\overline{\mathbf{5}})} \\
\lambda^{\tilde{\psi}_{1}(\overline{\mathbf{5}})-\tilde{\psi}_{2}(\overline{\mathbf{5}})} & 1 & \lambda^{\tilde{\psi}_{2}(\overline{\mathbf{5}})-\tilde{t}(\overline{\mathbf{5}})} \\
\lambda^{\tilde{\psi}_{1}(\overline{\mathbf{5}})-\tilde{t}(\overline{\mathbf{5}})} & \lambda^{\tilde{\psi}_{2}(\overline{\mathbf{5}})-\tilde{t}(\overline{\mathbf{5}})} & 1
\end{array}\right)
$$


The concrete model in Tables 2 and 3 gives

$$
\begin{aligned}
Y_{u} & \sim\left(\begin{array}{ccc}
\lambda^{\frac{77}{6}} & \lambda^{10} & \lambda^{\frac{77}{12}} \\
\lambda^{10} & \lambda^{\frac{43}{6}} & \lambda^{\frac{43}{12}} \\
\lambda^{\frac{77}{12}} & \lambda^{\frac{43}{12}} & 1
\end{array}\right), Y_{d} \sim Y_{e}^{T} \sim\left(\begin{array}{ccc}
\lambda^{\frac{79}{6}} & \lambda^{\frac{31}{3}} & \lambda^{10} \\
\lambda^{\frac{31}{3}} & \lambda^{\frac{15}{2}} & \lambda^{\frac{43}{6}} \\
\lambda^{\frac{27}{4}} & \lambda^{\frac{47}{12}} & \lambda^{\frac{43}{12}}
\end{array}\right), \\
M_{\nu} & \sim \lambda^{-\frac{3}{2}}\left(\begin{array}{ccc}
\lambda^{\frac{19}{3}} & \lambda^{\frac{7}{2}} & \lambda^{\frac{19}{6}} \\
\lambda^{\frac{7}{2}} & \lambda^{\frac{2}{3}} & \lambda^{\frac{1}{3}} \\
\lambda^{\frac{19}{6}} & \lambda^{\frac{1}{3}} & 1
\end{array}\right) \frac{\left\langle h_{u}\right\rangle^{2} \eta^{2}}{\Lambda},
\end{aligned}
$$

from which the CKM matrix and MNS matrix are obtained by

$$
V_{C K M} \sim\left(\begin{array}{ccc}
1 & \lambda^{\frac{17}{6}} & \lambda^{\frac{77}{12}} \\
\lambda^{\frac{17}{6}} & 1 & \lambda^{\frac{43}{12}} \\
\lambda^{\frac{77}{12}} & \lambda^{\frac{43}{12}} & 1
\end{array}\right), V_{M N S} \sim\left(\begin{array}{ccc}
1 & \lambda^{\frac{17}{6}} & \lambda^{\frac{19}{6}} \\
\lambda^{\frac{17}{6}} & 1 & \lambda^{\frac{1}{3}} \\
\lambda^{\frac{19}{6}} & \lambda^{\frac{1}{3}} & 1
\end{array}\right)
$$

Taking $\lambda \sim 0.5$ gives reasonable values for quark and lepton masses and mixings. This leads to a bit small solar neutrino mixing angle, but a cancellation may make it larger, and the bi-large neutrino mixings can be obtained.

Unfortunately, the FCNC processes are not sufficiently suppressed in this model. Actually, the parameters $\delta_{\mathbf{1 0}}$ and $\delta_{\overline{\mathbf{5}}}$ are estimated as

$$
\begin{aligned}
\delta_{10} & \sim V_{C K M}^{\dagger}\left(\begin{array}{ccc}
\lambda^{\frac{4}{3}} & \lambda^{\frac{25}{6}} & \lambda^{\frac{77}{12}} \\
\lambda^{\frac{25}{6}} & \lambda^{\frac{4}{3}} & \lambda^{\frac{113}{12}} \\
\lambda^{\frac{77}{12}} & \lambda^{\frac{113}{12}} & 1
\end{array}\right) V_{C K M} \sim\left(\begin{array}{ccc}
\lambda^{\frac{4}{3}} & \lambda^{\frac{25}{6}} & \lambda^{\frac{77}{12}} \\
\lambda^{\frac{25}{6}} & \lambda^{\frac{4}{3}} & \lambda^{\frac{43}{12}} \\
\lambda^{\frac{77}{12}} & \lambda^{\frac{43}{12}} & 1
\end{array}\right), \\
\delta_{\overline{5}} & \sim V_{M N S}^{\dagger}\left(\begin{array}{ccc}
\lambda^{\frac{4}{3}} & \lambda^{\frac{17}{6}} & \lambda^{\frac{11}{3}} \\
\lambda^{\frac{17}{6}} & \lambda^{\frac{4}{3}} & \lambda^{\frac{13}{6}} \\
\lambda^{\frac{11}{3}} & \lambda^{\frac{13}{6}} & 1
\end{array}\right) V_{M N S} \sim\left(\begin{array}{ccc}
\lambda^{\frac{4}{3}} & \lambda^{\frac{17}{6}} & \lambda^{\frac{19}{6}} \\
\lambda^{\frac{17}{6}} & \lambda^{\frac{2}{3}} & \lambda^{\frac{1}{3}} \\
\lambda^{\frac{19}{6}} & \lambda^{\frac{1}{3}} & 1
\end{array}\right),
\end{aligned}
$$

which lead to too large FCNC processes when $\lambda \sim 0.5$. This is mainly because large mixings in $V_{M N S}$ transform the differences $\tilde{m}_{3}-\tilde{m}_{i}(i=1,2)$ to the other mixings in the paramters $\delta_{\text {bar5 }}$ and because the $S U(2)_{H}$ breaking scale, $\left|V_{C}\right|=\left|V_{\bar{C}}\right|=\left|V_{B}\right| \sim \lambda^{\frac{2}{3}} \sim$ $(0.22)^{\frac{1}{3}}$, is not so small to suppress the FCNC processes.

\section{4 horizontal symmetry in $E_{6}$ Higgs sectors}

In this section, we investigate $E_{6}$ models with an anomalous $U(1)_{A}$ gauge symmetry whose Higgs sectors have non-trivial quantum numbers of the horizontal symmetry, $S U(2)_{H}$ or $S U(3)_{H}$. Since $E_{6}$ contains $S U(2)_{E}$, these model may realize well-suppressed FCNC processes as suggested in 42 . In this sence, $E_{6}$ models are more promising than $S O(10)$ model considered in the previous section. Unfortunately, however, if both $E_{6}$ and the horizontal symmetry is simultaneously broken, it is anticipated to be difficult to obtain realistic models in which the FCNC processes are sufficiently suppressed. The point is as follows. In order to suppress sufficiently the FCNC processes by the horizontal symmetry, the scale at which the horizontal symmetry is broken should be smaller than $\lambda^{2}$. ( In this 
Table 4: The Higgs content of $E_{6} \times U(1)_{A}$ model of Ref. [6]: The symbols \pm denote an additional $Z_{2}$ parity. Here, the composite operator $\bar{\Phi} \Phi$ plays the role of the FN field $\Theta$.

\begin{tabular}{|c|c|c|}
\hline & non-vanishing VEV & vanishing VEV \\
\hline $\mathbf{7 8}$ & $A(a=-1,-)$ & $A^{\prime}\left(a^{\prime}=4,-\right)$ \\
$\mathbf{2 7}$ & $\Phi(\phi=-3,+) C(c=-6,+)$ & $C^{\prime}\left(c^{\prime}=7,-\right)$ \\
$\overline{\mathbf{2 7}}$ & $\bar{\Phi}(\bar{\phi}=2,+) \bar{C}(\bar{c}=-2,+)$ & $\bar{C}^{\prime}\left(\bar{c}^{\prime}=8,-\right)$ \\
$\mathbf{1}$ & $Z_{i}\left(z_{i}=-2,-\right)(i=1,2,3)$ & \\
\hline
\end{tabular}

Table 5: The Higgs content of $E_{6} \times U(1)_{A}$ model of Ref.[7]: The symbols \pm denote an additional $Z_{2}$ symmetry.

\begin{tabular}{|c|c|c|}
\hline & non-vanishing VEV & vanishing VEV \\
\hline $\mathbf{7 8}$ & $A(a=-1,+)$ & $A^{\prime}\left(a^{\prime}=5,+\right)$ \\
$\mathbf{2 7}$ & $\Phi(\phi=-3,+)$ & $C^{\prime}\left(c^{\prime}=6,-\right)$ \\
$\overline{\mathbf{2 7}}$ & $\bar{C}(\bar{c}=0,-)$ & $\bar{\Phi}^{\prime}\left(\bar{c}^{\prime}=5,+\right)$ \\
$\mathbf{1}$ & $\Theta(\theta=-1,+) Z_{i}\left(z_{i}=-1,-\right)(i=1,2)$ & \\
\hline
\end{tabular}

section, we take $\lambda \sim \sin \theta_{C} \sim 0.22$, and we do not fix the anomalous $U(1)_{A}$ charge of the FN field to -1 but $\langle\Theta\rangle \sim \lambda^{\theta}$.) Generically, In GUT models with an anomalous $U(1)_{A}$ symmetry, it is difficult to obtain a smaller $E_{6}$ breaking scale than $\lambda^{2}$ [6] [7. Therefore, if both $E_{6}$ and the horizontal symmetry are broken by a VEV of a single field, that is, both the symmetries are broken at the same scale, then the suppression of the FCNC processes does not become sufficient.

After a brief review of the $E_{6}$ models with an anomalous $U(1)_{A}$ symmetry and without a horizontal symmetry proposed before, we examine the possibility of the $S U(2)_{H}$ horizontal symmetry in $\$ 4.2$ and of the $S U(3)_{H}$ horizontal symmetry in $\$ 4.3$.

\section{1 $\quad E_{6}$ model without horizontal symmetry}

We have proposed two types of $E_{6}$ Higgs sector in Refs. [6] and [7]. A typical charge assignment for each model is displayed in Table 4 and Table 5, respectively. The nonvanishing VEVs are given as

$$
\begin{aligned}
\left\langle\mathbf{4 5}_{\mathbf{A}}\right\rangle & \sim \lambda^{-a}, \\
\left\langle\mathbf{1}_{\mathbf{\Phi}}\right\rangle=\left\langle\mathbf{1}_{\overline{\mathbf{\Phi}}}\right\rangle & \sim \lambda^{-\frac{1}{2}(\phi+\bar{\phi})}, \\
\left\langle\mathbf{1 6}_{\mathbf{C}}\right\rangle=\left\langle\overline{\mathbf{1 6}}_{\overline{\mathbf{C}}}\right\rangle & \sim \lambda^{-\frac{1}{2}(c+\bar{c})},
\end{aligned}
$$

in the former model and

$$
\begin{aligned}
\left\langle\mathbf{4 5}_{A}\right\rangle & \sim \lambda^{-a}, \\
\left\langle\overline{\mathbf{1 6}}_{\bar{C}}\right\rangle \sim\left\langle\mathbf{1 6}_{A}\right\rangle \sim\left\langle\mathbf{1}_{\Phi}\right\rangle & \sim \lambda^{-\frac{1}{3}(\bar{c}+a+\phi)}, \\
\left\langle\mathbf{1}_{\bar{C}}\right\rangle \sim\left\langle\mathbf{1 6}_{\Phi}\right\rangle & \sim \lambda^{-\frac{1}{3}(-a+2 \bar{c}+2 \phi)},
\end{aligned}
$$


in the latter model. The correspondence to the $S O(10)$ model in $\$ 3.1$ is understood by the decomposition of $E_{6}$ representations,

$$
\begin{aligned}
& 78 \rightarrow 45+1+16+\overline{16} \\
& 27 \rightarrow 16+10+1
\end{aligned}
$$

in terms of $S O(10)$ representations. The VEVs along the $\mathbf{1}$ components of $\mathbf{2 7}$ Higgs break $E_{6}$ into $S O(10)$. The adjoint Higgs, $A$, of the $S O(10)$ model is embedded into the adjoint Higgs, $A$, in each model. The spinor Higgs, $C(\bar{C})$, is embedded into the field that have an non-vanishing VEV along the $\mathbf{1 6}(\overline{\mathbf{1 6}})$ component. And the MSSM doublet Higgs are embedded into $\Phi$ mainly.

As mentioned below, we use the former type to unify the Higgs sectors for $E_{6}$ breaking and for horizontal symmetry breaking. Thus let us concentrate on the former type. Here, we also assume the VEV relations Eq.(9) and thus effective charge is well-defined as

$$
\tilde{x}=x+c_{V} \Delta c+c_{V^{\prime}} \Delta \phi
$$

where $c_{V}$ and $c_{V^{\prime}}$ are $U(1)_{V}$ and $U(1)_{V^{\prime}}$ charges of the field $X$ which are normalized by the charges of the component with non-vanishing VEVs $\langle C\rangle$ and $\langle\Phi\rangle$, respectively. $\Delta c$ and $\Delta \phi$ are determined by the relations

$$
\langle C\rangle \sim \lambda^{-\tilde{c}}, \quad\langle\Phi\rangle \sim \lambda^{-\tilde{\phi}} .
$$

This assumption on the VEV relations (9) is natural if we introduce the generic interaction as comented in $\$ 3.1$ Then, the non-vanishing VEVs are determined by the superpotential that is linear in the field with vanishing VEV,

$$
W=W_{A^{\prime}}+W_{C^{\prime}}+W_{\bar{C}^{\prime}} .
$$

$W_{C^{\prime}}$ and $W_{\bar{C}^{\prime}}$ play similar roles as in the $S O(10)$ model. In $W_{A^{\prime}}$, we have to introduce a term $\bar{\Phi} A^{\prime} A^{3} \Phi$ or $A^{\prime} A^{5}$, since the term $A^{\prime} A^{3}$ does not contain the term, $\left(\mathbf{4 5}_{A^{\prime}} \mathbf{4} \mathbf{4}_{A}\right)_{\mathbf{5 4}}\left(\mathbf{4 5 _ { A } ^ { 2 }}\right)_{\mathbf{5 4}}$, which is required to obtain the DW form of $\mathbf{4 5} \mathbf{5}_{A}$ in a natural way. And the term, $\bar{\Phi} A^{\prime} A \Phi$, is also required, because without this term, some components of $A$ can not obtain superheavy masses due to an accidental symmetry in the superpotential that determine the VEVs, $\langle A\rangle,\langle\Phi\rangle$ and $\langle\bar{\Phi}\rangle$, namely they become Pseudo Nambu-Goldstone (PNG) modes. However, the larger $a^{\prime}+a$ results in the lighter mass spectrum for superheavy modes which make the gauge couplings evaluated at the cutoff scale stronger. If we take $a^{\prime}+a>4$, the gauge couplings go to the non-perturbative region in most part of the parameter space. Thus, we cannot take $\phi+\bar{\phi}$ so small, and therefore $\langle\Phi\rangle$ and $\langle\bar{\Phi}\rangle$ cannot be so small. As for the mass spectrum of this Higgs sector, we need the term, $\bar{\Phi} \bar{\Phi} \bar{C}$, to give the effective mass term, $\left\langle\mathbf{1}_{\bar{\Phi}}\right\rangle \mathbf{1 0}_{\bar{\Phi}} \mathbf{1 0}_{\bar{C}}$. This term is essential to avoid unwanted light modes because one pair of $\mathbf{2 7}-\overline{\mathbf{2 7}}$ Higgs can not obtain mass with the primed field in this Higgs content while one pair of 16-16 components can obtain mass of the symmetry breaking scale through the Higgs mechanism.

In the quark and lepton sector, we introduce three fundamental matters, $\Psi_{i}(\mathbf{2 7})(i=$ $1,2,3)$, and their $U(1)_{A}$ charges are taken as $\psi_{1}-\psi_{2} \sim 1$ and $\psi_{2}-\psi_{3} \sim 2$ to reproduce the CKM mixings. The three fields, $\Psi_{i}$, include three 5 s and six $\overline{\mathbf{5}}$ s of $S U(5)$. They have mass 
terms through the two types of interaction $\lambda^{\psi_{i}+\psi_{j}+\phi} \Psi_{i} \Psi_{j} \Phi$ and $\lambda^{\psi_{i}+\psi_{j}+c} \Psi_{i} \Psi_{j} C$, yielding a $3 \times 6$ mass matrix,

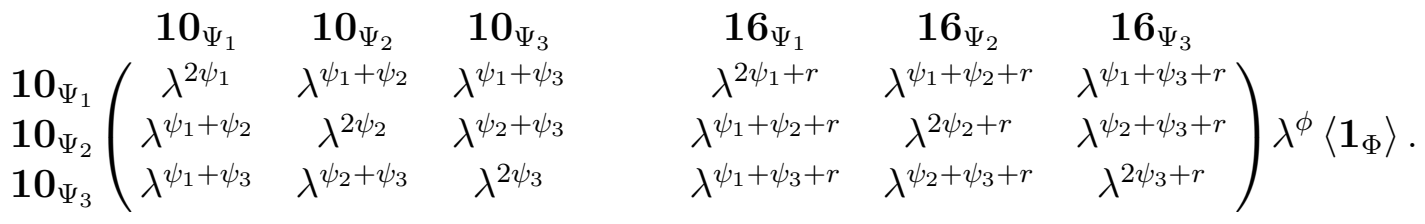

Here, for simplicity, we assume that the SUSY-zero mechanism does not forbit any elements. However, even if some of the elements of $3 \times 3$ matrix of $\mathbf{1 0}_{\Psi_{i}}$ and $\mathbf{1 6}{\Psi_{\Psi_{i}}}_{\text {vanish }}$ by the SUSY-zero mechanism, the following arguments can be applied unless the ranks of the $3 \times 3$ matrix is reduced. Here, the parameter $r$ is defined by $\lambda^{r} \equiv \frac{\lambda^{\phi}\langle\Phi\rangle}{\lambda^{c}\langle C\rangle}$. These mass terms make three pairs of $\mathbf{5}$ and $\overline{\mathbf{5}}$ superheavy, while three $\overline{\mathbf{5}}$ s remain massless. Providing $0 \leq r \leq \psi_{1}-\psi_{3}$, these three modes can be written as

$$
\begin{aligned}
& \overline{\mathbf{5}}_{1} \sim \mathbf{1 6} \mathbf{6}_{\Psi_{1}}+\lambda^{\psi_{1}-\psi_{3}} \mathbf{1 6}{\mathbf{\Psi _ { 3 }}}+\lambda^{\psi_{1}-\psi_{2}+r} \mathbf{1 0}_{\Psi_{2}}+\lambda^{\psi_{1}-\psi_{3}+r} \mathbf{1 0}_{\Psi_{3}}, \\
& \overline{\mathbf{5}}_{2} \sim \mathbf{1 0}{\Psi_{1}}+\lambda^{\psi_{1}-\psi_{3}-r} \mathbf{1 6}{\Psi_{3}}+\lambda^{\psi_{1}-\psi_{2}} \mathbf{1 0} 0_{\Psi_{2}}+\lambda^{\psi_{1}-\psi_{3}} \mathbf{1 0}_{\Psi_{3}} \text {, } \\
& \overline{\mathbf{5}}_{3} \sim \mathbf{1 6}{\mathbf{\Psi _ { 2 }}}_{2}+\lambda^{\psi_{2}-\psi_{3}} \mathbf{1 6} \mathbf{6}_{\Psi_{3}}+\lambda^{r} \mathbf{1 0}{\mathbf{\Psi _ { 2 }}}_{2}+\lambda^{\psi_{2}-\psi_{3}+r} \mathbf{1 0}_{\Psi_{3}} \text {, }
\end{aligned}
$$

where we use a base in which each light mode includes no other main modes. From these mixing, we can find the Yukawa matrices of the down-type quarks and the charged leptons are given as

$$
Y_{d} \sim Y_{e}^{T} \sim\left(\begin{array}{ccc}
\lambda^{2 \psi_{1}-\psi_{2}-\psi_{3}} & {\left[\lambda^{2 \psi_{1}-\psi_{2}-\psi_{3}-r}\right]} & {\left[\lambda^{\psi_{1}-\psi_{3}}\right]} \\
{\left[\lambda^{\psi_{1}-\psi_{3}}\right]} & {\left[\lambda_{1}-\psi_{3}-r\right]} & \lambda^{\psi_{2}-\psi_{3}} \\
\lambda^{\psi_{1}-\psi_{2}} & \lambda^{\psi_{1}-\psi_{2}-r} & 1
\end{array}\right) \lambda^{\psi_{2}-\psi_{3}}
$$

if we set $2 \psi_{3}+\phi=0$ to reproduce the $\mathcal{O}(1)$ top Yukawa coupling. Note that although in the $3 \times 6$ matrix (62) the $S U(2)_{R}$ breaking VEV, $\langle C\rangle$, appears through the Yukawa interaction, $\Psi_{i} \Psi_{j} C$, this breaking effect is not sufficient to produce the Cabibbo mixing. Actually, unless there is the $S U(2)_{R}$ breaking in the Yukawa couplings of $\Psi_{i} \Psi_{j} \Phi$ and $\Psi_{i} \Psi_{j} C$ or in MSSM higgs mixings as in $S O(10)$ case (24), the components with the parenthesis in the matrix (66) become smaller in the basis where the Yukawa matrix of the up-type quarks is diagonalized. Then, the Cabibbo angle becomes smaller than the naively expected value, $\lambda^{\psi_{1}-\psi_{2}}$. Therefore, the $S U(2)_{R}$ breaking effect have to appear at least either of in the Yukawa couplings or in the MSSM higgs mixings. Then, the CKM matrix is obtained as

$$
V_{\mathrm{CKM}} \sim\left(\begin{array}{ccc}
1 & \lambda^{\psi_{1}-\psi_{2}} & \lambda^{\psi_{1}-\psi_{3}} \\
\lambda^{\psi_{1}-\psi_{2}} & 1 & \lambda^{\psi_{2}-\psi_{3}} \\
\lambda^{\psi_{1}-\psi_{3}} & \lambda^{\psi_{2}-\psi_{3}} & 1
\end{array}\right) \sim\left(\begin{array}{ccc}
1 & \lambda & \lambda^{3} \\
\lambda & 1 & \lambda^{2} \\
\lambda^{3} & \lambda^{2} & 1
\end{array}\right) .
$$

Thus, the CKM matrix are determined by the differnce of the $U(1)_{A}$ charges, $\psi_{i}-\psi_{j}$, and therefore, by the difference of the effective $U(1)_{A}$ charges, $\tilde{\psi}_{i}(\mathbf{1 6}, \mathbf{1 0})-\tilde{\psi}_{j}(\mathbf{1 6}, \mathbf{1 0})=\psi_{i}-$ $\psi_{j}$. Here, we denote the representations of $S O(10)$ and $S U(5)$, explicitly. The MNS matrix 
is also determined by the difference of the effective charges, $\tilde{\psi}_{1}(\mathbf{1 6}, \overline{\mathbf{5}})-\tilde{\psi}_{1}(\mathbf{1 0}, \overline{\mathbf{5}})=r$ and $\tilde{\psi}_{1}(\mathbf{1 6}, \overline{\mathbf{5}})-\tilde{\psi}_{2}(\mathbf{1 6}, \overline{\mathbf{5}})=\psi_{1}-\psi_{2}$ as

$$
V_{\mathrm{MNS}} \sim\left(\begin{array}{ccc}
1 & \lambda^{r} & \lambda^{\psi_{1}-\psi_{2}} \\
\lambda^{r} & 1 & \lambda^{\psi_{1}-r-\psi_{2}} \\
\lambda^{\psi_{1}-\psi_{2}} & \lambda^{\psi_{1}-r-\psi_{2}} & 1
\end{array}\right) \sim\left(\begin{array}{ccc}
1 & \lambda^{r} & \lambda \\
\lambda^{r} & 1 & \lambda^{1-r} \\
\lambda & \lambda^{1-r} & 1
\end{array}\right) .
$$

Because the mass of the light neutrinos is given from the operators, $L_{i} L_{j} H_{u}^{2}$, we can estimate neutrino mass matrix as

$$
M_{\nu} \sim\left(\begin{array}{ccc}
\lambda^{2\left(\psi_{1}-\psi_{2}\right)} & \lambda^{2 \psi_{1}-r-2 \psi_{2}} & \lambda^{\psi_{1}-\psi_{2}} \\
\lambda^{2 \psi_{1}-r-2 \psi_{2}} & \lambda^{2\left(\psi_{1}-r-\psi_{2}\right)} & \lambda^{\psi_{1}-r-\psi_{2}} \\
\lambda^{\psi_{1}-\psi_{2}} & \lambda^{\psi_{1}-r-\psi_{2}} & 1
\end{array}\right) \lambda^{2\left(\psi_{2}+\phi-r+\Delta \phi\right)} \frac{\eta^{2}\left\langle H_{u}^{2}\right\rangle}{\Lambda},
$$

where $\tilde{\psi}_{2}(\mathbf{1 6}, \overline{\mathbf{5}})+\tilde{\phi}(\mathbf{1 0}, \mathbf{5})=\psi_{2}+\phi-r+\Delta \phi$. Let us introduce a parameter $l$ that parameterize the mass of the heaviest light neutrino, $m_{\nu_{3}}$, as $-(l+5) \equiv 2 \psi_{2}+2(\phi-r+\Delta \phi)$, namely

$$
\lambda^{(l+5)} \sim \frac{\eta^{2}\left\langle H_{u}^{2}\right\rangle}{m_{\nu_{3}} \Lambda} .
$$

In order to explain the atmospheric neutrino experiments [13], $l$ should be around $-1--4$.

Finally, let us comment on the gauge coupling unification. In Ref. 18, it is shown that the success of the gauge coupling unification in the minimal SUSY $S U(5)$ model can completely be reproduced in the scenario of GUT with an anomalous $U(1)_{A}$ gauge symmetry if the VEV relation (9) holds, although the mass spectrum of superheavy fields does not respect the $S U(5)$ gauge symmetry. In the latter scenario, the effective mass of the color triplet partners of the MSSM doublet Higgs should be around the usual GUT scale $\Lambda_{G} \sim 2 \times 10^{16} \mathrm{GeV}$, if the contribution from $\mathcal{O}(1)$ coefficients that we have omitted is negletcted. This condition can be expressed as

$$
m_{C}^{\mathrm{eff}} \sim \lambda^{2 \phi+\Delta \phi} \sim 1 .
$$

Because the cutoff scale in the scenario is around the usual GUT scale, $\Lambda_{G}$, this condition may lead to too rapid proton decay via dimension 5 operators. However, in the scenario, the masses of superheavy fields are determined by the anomalous $U(1)_{A}$ charges but still have the ambiguity of the $\mathcal{O}(1)$ coefficients. Because the number of the superheavy fields is large, the contribution of the $\mathcal{O}(1)$ coefficients cannot be neglected. Actually, the ambiguity due to the $\mathcal{O}(1)$ coefficients makes larger $m_{C}^{\text {eff }}$ possible, and indeed we push $m_{C}^{\text {eff }}$ larger to suppress the proton decay via dimension 5 operators. But larger $m_{C}^{\text {eff }}$ requires a larger ambiguity which seems less natural. Thus, as long as the sufficient suppression of the proton decay is achieved, a smaller $m_{C}^{\text {eff }}$, which is realized by larger $\phi$ and larger $\Delta \phi$, is preferred for the gauge coupling unification.

\section{$4.2 \quad E_{6} \times S U(2)_{H}$}

Since the matter sector can be unified in terms of the $S U(2)_{H}$ horizontal symmetry as in 42 , the next task is to unify the Higgs sector. Motivated by the decomposition 
$E_{8} \supset E_{6} \times S U(3)_{H} \supset E_{6} \times S U(2)_{H}$, under which $\mathbf{2 4 8}$ of $E_{8}$ is decomposed as

$$
\begin{aligned}
248 & \rightarrow(78,1)+(1,8)+(27,3)+(\overline{27}, \overline{3}) \\
& \rightarrow(78,1)+(1,3+2+2+1)+(27,2+1)+(\overline{27}, 2+1),
\end{aligned}
$$

we assign non-trivial representation of the horizontal symmetry only to $\mathbf{2 7}, \overline{\mathbf{2 7}}$ and $\mathbf{1}$ Higgs. By the way, in the matter sector, two $\mathbf{2 7}$ for the 1st and 2nd generations are treated as a doublet, and the difference of their effective charges should correspond to the Cabibbo angle, $\widetilde{\psi}_{1}-\widetilde{\psi}_{2} \sim 1$. This means the difference of the effective charges of two components of doublets should be also around 1 if the effective charge is well-defined ${ }^{2}$. In the Table 1 , we introduce two $\mathbf{2 7}\left(\Phi, C^{\prime}\right)$ and two $\overline{\mathbf{2 7}}\left(\bar{C}, \bar{C}^{\prime}\right)$, where the primed fields have positive charges and the unprimed fields have negative charges. And the difference of anomalous $U(1)_{A}$ charges of each two fields is much larger than 1 . Thus, it is difficult to unify the Higgs sector of the Table ${ }^{3}{ }^{3}$. And thus, we concentrate on the Higgs sector of the Table 4, which contain

$$
\begin{aligned}
& 78: A, \quad A^{\prime} \\
& 27 \text { : } \Phi, C, C^{\prime} \\
& \overline{27}: \bar{\Phi}, \bar{C}, \bar{C}^{\prime}
\end{aligned}
$$

and singlets. By the same reason as mentioned in the previous footnote, it is difficult to embed the primed fields into a doublet if we aim to suppress the FCNC processes not assuming the universal soft mass. If we take $(\Phi, C)$ as a doublet under $S U(2)_{H}$, the Yukawa interaction for the top quark, $\Psi_{3} \Psi_{3} \Phi$, is forbidden by the horizontal symmetry. Thus the remaining possibility is to embed $\bar{\Phi}$ and $\bar{C}$ into a doublet as $\bar{C}_{a}=(\bar{\Phi}, \bar{C})$.

The Higgs content we consider below is summarized in Table 6. For simplicity, we

Table 6: The Higgs content of $E_{6} \times S U(2)_{H} \times U(1)_{A}$ models except for singlets: Here $S U(2)_{H}$ doublets are denoted by the index $a$. All the non-vanishing VEVs are shown, and their magnitudes are formally written by introducing parameters $\Delta \phi$ etc.. One or more discreate symmetries are introduced according to need.

\begin{tabular}{|c|c|c|}
\hline & non-vanishing VEV & vanishing VEV \\
\hline $\mathbf{7 8}$ & $A\left(\left\langle\mathbf{4} \mathbf{5}_{A}\right\rangle \sim \lambda^{-a}\right)$ & $A^{\prime}$ \\
$\mathbf{2 7}$ & $\Phi\left(\left\langle\mathbf{1}_{\Phi}\right\rangle \sim \lambda^{-(\phi-\Delta \phi)}\right), C\left(\left\langle\mathbf{1 6}_{C}\right\rangle \sim \lambda^{-(c-\Delta c)}\right)$ & $C^{\prime}$ \\
$\overline{\mathbf{2 7}}$ & $\bar{C}_{a}\left(\left\langle\mathbf{1}_{\left.\left.\bar{C}_{1}\right\rangle \sim \lambda^{-\left(\bar{c}+\Delta \phi+\Delta f_{c}\right)},\left\langle\overline{\mathbf{1 6}}_{\bar{C}_{2}}\right\rangle \sim \lambda^{-\left(\bar{c}+\Delta c-\Delta \bar{f}_{c}\right)}\right)} \bar{C}^{\prime}\right.\right.$ \\
$\mathbf{1}$ & $\bar{F}_{a}\left(\left\langle\bar{F}_{1}\right\rangle \sim \lambda^{-(\bar{f}+\Delta \bar{f})}\right), F_{a}\left(\left\langle F_{2}\right\rangle \sim \lambda^{-(f-\Delta f)}\right)$ & \\
\hline
\end{tabular}

assume that any component fields other than shown in Table [6 have vanishing VEVs.

\footnotetext{
${ }^{2}$ Later, we consider models where the effective charge is not well-defined. But as in the analysis, if the ill-definedness is small, then the following discussion can be applied.

${ }^{3}$ If we take $\tilde{\psi}_{1}-\tilde{\psi}_{2} \gg 1$, which corresponds $\lambda>\sin \theta_{C}$, we may obtain a suitable model which has realistic quark and lepton masses and mixings as in the previous $S O(10)$ case. However, it must be difficult for the horizontal symmetry to suppress the FCNC processes sufficiently. Of course, if the universality of sfermion masses are guaranteed by some other mechanism, such models can be realistic. But we do not examine such possibility here.
} 
If three GUT and horizontal gauge singlets have non-vanishing VEVs as in the VEV relations (9) from three $F$-flatness conditions, for example,

$$
\begin{aligned}
\Phi \bar{C} F & \sim \lambda^{-(\phi+\bar{c}+f)} \equiv \lambda^{-3 k}, \\
C \bar{C} \bar{F} & \sim \lambda^{-(c+\bar{c}+\bar{f})}, \\
F \bar{F} & \sim \lambda^{-(f+\bar{f})},
\end{aligned}
$$

relations $\Delta f=\Delta \bar{f}=\Delta f_{c}=\Delta \bar{f}_{c}$ are obtained. Once these relations are fixed by three $F$-flatness conditions, the VEVs of the other singlet operators that have non-vanishing VEVs automatically satisfy the VEV relations (92). When some of the non-vanishing VEVs do not satisfy the VEV relations (9), generally, these $\Delta f_{\mathrm{s}}$ have different values. Such models will be discussed later, in which the effective charge can not be well-defined. But for the moments, let us assume Eqs.(75)-(77) hold. In addition to the three relations, three D-flatness conditions

$$
\begin{aligned}
\left|\mathbf{1}_{\phi}\right|^{2} & =\left|\mathbf{1}_{\bar{C}_{1}}\right|^{2}, \\
\left|\mathbf{1 6}_{C}\right|^{2} & =\left|\overline{\mathbf{1 6}}_{\bar{C}_{2}}\right|^{2}, \\
\left|\mathbf{1}_{\bar{C}_{1}}\right|^{2}+\left|\bar{F}_{1}\right|^{2} & =\left|\overline{\mathbf{1 6}}_{\bar{C}_{2}}\right|^{2}+\left|F_{2}\right|^{2}
\end{aligned}
$$

determine three parameters, $\Delta \phi, \Delta c$ and $\Delta f$, in terms of the anomalous $U(1)$ charges. Roughly, there are four possible cases as follows:

1. $\mathbf{1}_{\bar{C}_{1}} \sim \overline{\mathbf{1 6}}_{\bar{C}_{2}} \geq \bar{F}_{1}, F_{2}$

2. $\bar{F}_{1} \sim \overline{\mathbf{1 6}}_{\bar{C}_{2}} \geq \mathbf{1}_{\bar{C}_{1}}, F_{2}$

3. $\bar{F}_{1} \sim F_{2} \geq \mathbf{1}_{\bar{C}_{1}}, \overline{\mathbf{1 6}}_{\bar{C}_{2}}$

4. $\mathbf{1}_{\bar{C}_{1}} \sim F_{2} \geq \bar{F}_{1}, \overline{\mathbf{1 6}}_{\bar{C}_{2}}$

As for the 2nd and 3rd case, the horizontal breaking scale is larger than the GUT breaking scale $\langle\mathbf{1}\rangle$. As discussed in $\langle\mathbf{1}\rangle$ does not seem so small as $\lambda^{2}$. Therefore, $S U(2)_{H}$ breaking scale larger than $\langle\mathbf{1}\rangle$ is not sufficient for the suppression of the FCNC processes. For simplicity, we concentrate on the 4th case in the following discussion, but a similar discussion can be applied to the other cases. In the 4th case,

$$
\begin{aligned}
\mathbf{1}_{\Phi}=\mathbf{1}_{\bar{C}_{1}} \sim F_{2} & \sim \lambda^{-k}, \\
\bar{F}_{1} & \sim \lambda^{-(f+\bar{f})+k}, \\
\mathbf{1 6}_{C}=\overline{\mathbf{1 6}}_{\bar{C}_{2}} & \sim \lambda^{\frac{1}{2}[-(c+\bar{c})+f-k]},
\end{aligned}
$$

in other words,

$$
\begin{aligned}
\Delta f & =\frac{2 f-\phi-\bar{c}}{3}=f-k, \\
\Delta \phi & =\frac{2 \phi-f-\bar{c}}{3}=\phi-k, \\
\Delta c & =\frac{c-\bar{c}+\Delta f}{2} .
\end{aligned}
$$


The condition for the 4 th case $\left(F_{2} \geq \bar{F}_{1}, \overline{\mathbf{1 6}}_{\bar{C}_{2}}\right)$ to be realized is given by

$$
0<-k \leq-\frac{1}{2}(f+\bar{f}),-c-\bar{c}+f
$$

which is also written as

$$
f<\Delta f \leq \frac{1}{2}(f-\bar{f}),-c-\bar{c}+2 f .
$$

In addition, as shown in $\$ 4.1$ the following conditions are required phenomenologically:

- The parameter $r$ for the neutrino mixings should be around $\frac{1}{2}-\frac{3}{2}$.

- The parameter $l$ for the neutrino mass scale should be around -1--4.

- In order to realize the DT splitting, $C^{\prime} A \Phi \Phi$ must be allowed, and $C^{\prime} A C \Phi$ must be forbidden.

- $\bar{C} F \bar{C} F \bar{C} \bar{F}$, which corresponds to $\bar{\Phi} \bar{\Phi} \bar{C}$ in 4.1 must be allowed in order to avoid undesired massless modes.

- In order to give mass to would-be PNG modes, $A^{\prime} A \Phi \bar{C} F$ must be allowed.

- For the gauge coupling unification, smaller effective mass of the colored Higgs, $m_{C}^{\text {eff }} \sim$ $\lambda^{2 \phi+\Delta \phi}$, is preferable. In the model displayed in Table 4. $m_{C}^{\text {eff }} \sim \lambda^{-8.5}$.

- In order to reproduce the realistic quark mass matrices, the $S U(2)_{R}$ symmetry must be broken in the Yukawa couplings. $S U(2)_{R}$ breaking VEVs $\langle C\rangle=\langle\bar{C}\rangle$ can be picked up through the SM Higgs mixing $\left(\bar{C}^{\prime} \bar{C} \bar{F} A \Phi^{2}\right.$ is required), or through higher dimensional interactions (for example, $\Psi \bar{C} C \Psi \bar{F} \Phi)^{4}$.

These conditions are rewritten in terms of the anomalous $U(1)$ charges as

$$
\begin{aligned}
& \frac{1}{2} \lesssim r=\frac{1}{2}(c-\phi)+\Delta f \lesssim \frac{3}{2} \\
& -1 \gtrsim l=-5-2\left(\psi-\Delta f-\psi_{3}\right)+\phi+2 \Delta c \gtrsim-4 \\
& c<\phi \\
& \bar{f} \geq-3 \bar{c}-2 f \\
& 0 \leq a^{\prime}+a+\phi+\bar{c}+f \geq 0 \\
& 2 \phi+\Delta \phi \gtrsim-8.5 \\
& 2 \psi+\phi+c+\bar{c}+\bar{f} \geq 0 \quad \text { or } \quad \bar{c}^{\prime} \geq-2 \phi-\bar{c}-\bar{f}-a,
\end{aligned}
$$

Note that the 1 st condition is not consistent with the $3 \mathrm{rd}$ conditions if $\tilde{\psi}_{1}-\tilde{\psi}_{2}=1$, that is, $\Delta f=\frac{1}{2}$ to reproduce the suitable value of the Cabibbo angle. There are three ways to avoid this inconsistency ${ }^{5}$.

\footnotetext{
${ }^{4}$ Although there are $S U(2)_{R}$ breaking effects in the $3 \times 6$ mass matrix of $\mathbf{5}-\overline{\mathbf{5}}$ components of $\Psi_{a}$ and $\Psi_{3}$, it is not sufficient to reproduce the realistic quark mixings 6 . Thus we need another source of $S U(2)_{R}$ breaking.

${ }^{5}$ As in the previous $S O(10)$ model, we can take $\tilde{\psi}_{1}-\tilde{\psi}_{2}>1$ to avoid this inconsistency. Because the atmospheric neutrino mixings tend to be rather small, here, we do not examine this possibility.
} 
1. To relax the 1st requirement.

For example, $r=\frac{1}{4}$ is not an unacceptable choice, although rather large ambiguity of $\mathcal{O}(1)$ coefficients are needed to reproduce the large atmospheric neutrino oscillation.

2. To set $c \geq \phi$ and introduce an additional discrete symmetry to forbid $C^{\prime} A C \Phi$. In this case, $r=\frac{1}{2}(c-\phi+2 \Delta f)$. If $c=\phi$ is taken, the relation $r=\frac{1}{2}$ is obtained.

3. To give up the effective charge.

In terms of the notation in Table [ $r$ is given by

$$
r=\Delta c-\Delta \phi=\frac{c-\phi+\Delta f_{c}+\Delta \bar{f}_{c}}{2} .
$$

Thus, if $\Delta f_{c}+\Delta \bar{f}_{c}>\psi_{1}-\psi_{2}$, sufficiently large $r$ can be obtained without imposing additional symmetries.

Along each strategy, we construct realistic models as follows.

\subsection{1 $c<\phi\left(r<\frac{1}{2}\right)$}

In this analysis, we fix the $\Delta f$ as $\frac{1}{2}$. The relation $r=\frac{1}{2}(c-\phi+1)$ indicates that the larger $c-\phi<0$ leads to larger $r<\frac{1}{2}$. Therefore, if $c-\phi$ is taken as the minus minimum unit of $U(1)_{A}$ charge, then $r$ becomes the closest value to $\frac{1}{2}$. Therefore, the smaller unit leads to the closer value of $r$ to $\frac{1}{2}$. Here, we introduce half integer $U(1)_{A}$ charges and take $\theta=-\frac{1}{2}$, which give $r=\frac{1}{4}$.

As noted before, in the vacuum 4 , the $S U(2)_{H}$ breaking scale is the same as the $E_{6}$ breaking scale, because the VEVs $\left\langle\mathbf{1}_{\Phi}\right\rangle=\left\langle\mathbf{1}_{\bar{C}_{1}}\right\rangle \sim\left\langle F_{2}\right\rangle \sim \lambda^{-k}$ break simultaneously $S U(2)_{H}$ and $E_{6}$. In order to suppress the FCNC processes, a smaller $S U(2)_{H}$ breaking scale is preferable, but on the other hand, a smaller $E_{6}$ breaking scale leads to a larger effective colored Higgs mass, which can spoil the success of the gauge coupling unification and/or result in the non-perturbative region of gauge couplings, as noted in 4.1 . Taking account of the above conflict, we take $k=-1$ here. Thus, the relation $k=f-\Delta f$ leads to $f=-\frac{1}{2}$.

Then, the condition for the vacuum structure 4 Eq.(87), and the condition (92) give a relation

$$
2 k-f \geq-3 \bar{c}-2 f,
$$

that is, $\bar{c} \geq \frac{5}{6}$. Under fixed $k$ and $f$, because $3 k=\bar{c}+f+\phi$, larger $\bar{c}$ leads to smaller $\phi$, and therefore a larger colored Higgs mass, which leads to less natural explanation for the success of the gauge coupling unification. Therefore, we adopt $\bar{c}=1$, which leads to $\phi=-\frac{7}{2}$ and $c=-4$.

Now, Eq. (87) and $\bar{f} \geq-3 \bar{c}-2 f$ lead to $-2 \leq \bar{f} \leq-\frac{3}{2}$. And we take $\bar{f}=-2$.

As for $a, a=-\frac{1}{2}$ and $a=-1$ are possible. The former yields relatively large FCNC processes because $\langle A\rangle$ breaks the $S U(2)_{E}$ symmetry which guarantees the universality of masses of three $\overline{\mathbf{5}}$ sfermion fields. Therefore, we take $a=-1$, though the gauge couplings may become in non-perturbative region.

Table 7 shows examples for the latter case. $\left(a^{\prime}, c^{\prime}, \bar{c}^{\prime}\right)$ are determined by the smallest values that allow $A^{\prime} A^{5}, A^{\prime} \Phi \bar{C} F, C^{\prime} A \Phi \Phi$ and $\bar{C}^{\prime} Z C$. We set $z$ the largest value which 
Table 7: Examples of the charge assignments for the 1st and 2nd strategies : Signs denote the additional $Z_{2}$ symmetry that play the same role as the $Z_{2}$ symmetry introduced in Table 4. This charge assignment yields $r=\frac{1}{2}+\frac{c-\phi}{2}$ and $l=-5-c$. Odd quarter integer charges of the matter fields $\left(\Psi_{3}, \Psi_{a}\right)$ guarantees that the $R$-parity is automatically conserved. When $c \geq \phi$, we impose an additional $Z_{2}$ symmetry and introduce a singlet field $Z_{C}$ to forbit $C^{\prime} A C \Phi$ while allowing $C^{\prime} A \Phi \Phi$.

\begin{tabular}{|c|c|c|}
\hline & non-vanishing VEV & vanishing VEV \\
\hline $\mathbf{7 8}$ & $A(a=-1 ;-)$ & $A^{\prime}\left(a^{\prime}=5 ;-\right)$ \\
$\mathbf{2 7}$ & $\Phi(\phi=-7 / 2 ;+), C(c=-4,-7 / 2,-3,-5 / 2 ;+)$ & $C^{\prime}\left(c^{\prime}=8 ;-\right)$ \\
& & $\Psi_{3}\left(\psi_{3}=7 / 4 ;+\right), \Psi_{a}(\psi=17 / 4 ;+)$ \\
$\overline{\mathbf{2 7}}$ & $\bar{C}_{a}(\bar{c}=1 ;+)$ & $\bar{C}^{\prime}\left(\bar{c}^{\prime}=11 / 2 ;-\right)$ \\
$\mathbf{1}$ & $\bar{F}_{a}(\bar{f}=-2 ;+), F_{a}(f=-1 / 2 ;+)$ & \\
& $\Theta(\theta=-1 / 2 ;+), Z_{i}\left(z_{i}=-3 / 2 ;-\right)$ & \\
& $Z_{C}\left(z_{C}=-,-1 / 2,-1,-3 / 2 ;+\right)$ & \\
\hline
\end{tabular}

forbids $C^{\prime} Z \Phi \Phi$. Here, the matter fields $\left(\Psi_{3}, \Psi_{a}\right)$ are also shown. From their charges, we can find $l=-1$ and $\Psi \Psi \Phi C \bar{C} \bar{F}$ is allowed, which introduces $S U(2)_{R}$ breaking in the Yukawa couplings. Note that only the matter fields have odd quarter integer charges, and therefore they always appear in pair, which guarantees the $R$-parity is automatically conserved. The effective colored Higgs mass is given as $\lambda^{-19 / 2}$. This value is not so much different from the value of the model in Table 4. And the parameter $\delta_{\mathbf{1 0}}$ and $\delta_{\overline{\mathbf{5}}}$ are estimated as

$$
\delta_{\mathbf{1 0}} \sim\left(\begin{array}{ccc}
\lambda^{2} & \lambda^{3} & \lambda^{3} \\
\lambda^{3} & \lambda^{2} & \lambda^{2} \\
\lambda^{3} & \lambda^{2} & 1
\end{array}\right), \quad \delta_{\overline{\mathbf{5}}} \sim\left(\begin{array}{ccc}
\lambda^{2} & \lambda^{2+r} & \lambda^{3} \\
\lambda^{2+r} & \lambda^{2} & \lambda^{3-r} \\
\lambda^{3} & \lambda^{3-r} & \lambda^{2}
\end{array}\right),
$$

which are obtained from the non-renormalizable interactions, for example,

$$
\int d^{4} \theta Z^{\dagger} Z\left[|\Psi F|^{2}+\left|\Psi F^{\dagger}\right|^{2}+\Psi^{\dagger} A^{2} \Psi\right]
$$

Off diagonal elements of $\delta$ become smaller than those in the previous $S O(10)$ model, though these are still too large to suppress the FCNC processes and we must require other mechanisms that suppresses the above non-renormalizable interactions with the spurion field $Z$ or that gives universal sfermion masses.

\subsection{2 $\phi \leq c\left(r \geq \frac{1}{2}\right)$}

Next, let us examine the 2nd strategy. With the aid of an additional discrete symmetry, we can forbit the interaction $C^{\prime} A C \Phi$ while allowing $C^{\prime} A \Phi \Phi$ even when $\phi \leq c$, which always leads to $r \geq \frac{1}{2}$. For example, consider another $Z_{2}$ symmetry that only $C$ and $Z_{C}$ have odd parity. Here $z_{C}<\phi-c$ is required to forbid $C^{\prime} A C \Phi Z_{C}$ and to allow $C^{\prime} A \Phi \Phi$.

In this analysis, we also introduce half-integer charges and fix the value of $\Delta f$ as $\frac{1}{2}$. Then, as in the previous strategy, we set $(k, f, \bar{c}, \phi, \bar{f}, a)=\left(-1,-\frac{1}{2}, 1,-\frac{7}{2},-2,-1\right)$. 
For these charges, Eq.(87) requires $c \leq k-\bar{c}+f=-\frac{5}{2}$, which leads to $c=-\frac{7}{2},-3,-\frac{5}{2}$. $\left(a^{\prime}, c^{\prime}, z\right)$ are also determined as in the previous strategy. We set $z_{C}$ as the largest negative value satisfying $\phi>c+z_{C}$, and $\bar{c}^{\prime}$ is determined to allow $\bar{C}^{\prime} Z C Z_{C}{ }^{6}$. Table [7 summarizes the charge assignments.

Here, the matter fields $\left(\Psi_{3}, \Psi_{a}\right)$ are also shown. From their charges, we can find $l=-3 / 2,-2,-5 / 2$ and that $\Psi \Psi \Phi C Z_{C} \bar{C} \bar{F}$ is allowed, which is important to introduce $S U(2)_{R}$ breaking in Yukawa couplings. Again, the $R$-parity is automatically conserved. The effective colored Higgs mass is given as $\lambda^{-19 / 2}$. And the parameter $\delta_{\mathbf{1 0}}$ and $\delta_{\overline{\mathbf{5}}}$ are given by the same expression as in Eqs.(97).

\subsection{3 $\Delta f \neq \Delta \bar{f}$}

Finally, we investigate the possibility where the effective charge is not well-defined. It means the VEV relation (9) does not hold generally. Although this relation is naturally expected to hold, the ambiguity of $\mathcal{O}(1)$ coefficients may shift the relation slightly. Here, we assume one of the three singlet operators in Eqs.(75) - (77) has smaller non-vanishing VEV than given in the equaitons because of a cancellation.

From the definition $\lambda^{r} \equiv \frac{\lambda^{\phi}\langle\Phi\rangle}{\lambda^{c}\langle C\rangle}$, we can see the smaller $\langle\mathbf{1 6}\rangle$ and/or the larger $\langle\mathbf{1}\rangle$ result in larger $r$. Thus, if the cancellation occurs in Eq.(75), smaller $r$ will be resulted. If the cancellation occurs in Eq.(76), $r$ will be larger. However, in this case, $S U(2)_{R}$ breaking scale becomes smaller than expected by the charges, that is, the $S U(2)_{R}$ breaking effects in Yukawa couplings become smaller, which results in unrealistic quark mass matrices. Therefore, we assume

$$
F \bar{F} \sim \epsilon \lambda^{-(f+\bar{f})}
$$

where $\epsilon \ll 1$, instead of Eq.(77). Eqs.(75) and (76) mean $\Delta f=\Delta f_{c}$ and $\Delta \bar{f}=\Delta \bar{f}_{c}$, and therefore $\epsilon \sim \lambda^{\Delta f-\Delta \bar{f}} \equiv \lambda^{\delta}$. $\epsilon \ll 1$ leads to $\Delta f>\Delta \bar{f}$. In this case, magnitudes of couplings in the low energy effective theory depend on which interactions exist, in contrast to the case where the effective charge is well-defined and the magnitudes are written by a simple sum of the effective charge. Let us illustrate this by using the Yukawa matrix, $\Psi \Psi \Phi$ as a concrete example. For simplicity, we set $\psi_{3}=n=-\phi / 2$ and $\psi=n+m$. Then the Yukawa matrix in terms of exponent becomes

$$
\begin{aligned}
2 m+\left\{\begin{array}{l}
2 \Delta f \\
\Delta f+\Delta \bar{f} \\
2 \Delta \bar{f}
\end{array}\right. & 2 m+\left\{\begin{array}{l}
\Delta f-\Delta \bar{f} \\
0 \\
-\Delta f+\Delta \bar{f}
\end{array}\right. \\
2 m+\left\{\begin{array}{l}
\Delta f-\Delta \bar{f} \\
0 \\
-\Delta f+\Delta \bar{f}
\end{array}\right. & 2 m+\left\{\begin{array}{l}
\Delta f \\
-2 \bar{f} \\
-\Delta f-\Delta \bar{f} \\
-2 \Delta f
\end{array}\right. \\
m+\left\{\begin{array}{l}
\Delta f \\
\Delta \bar{f}
\end{array}\right. & m+\left\{\begin{array}{l}
-\Delta \bar{f} \\
-\Delta f \\
-\Delta f
\end{array}\right.
\end{aligned}
$$

where the term, for example, $m-\Delta \bar{f}$ denotes the element of Yukawa matrix is order of $\lambda^{m-\Delta \bar{f}}$. Here, the exponents $-\Delta \bar{f},+\Delta f,-\Delta f$ and $+\Delta \bar{f}$ come from the VEVs $\langle\bar{F}\rangle \sim$

\footnotetext{
${ }^{6}$ Another choice is to assign odd parity to $\bar{C}$ and determine $\bar{c}^{\prime}$ so as to $\bar{C}^{\prime} Z C$ allowed. This choice is convenient for embedding the model into $E_{6} \times S U(3)_{H}$ model, and we consider this possibility later.
} 
$\lambda^{-\bar{f}-\Delta \bar{f}},\langle F\rangle \sim \lambda^{-f+\Delta f},\langle\Phi \bar{C}\rangle \sim \lambda^{-\phi-\bar{c}-\Delta f}$ and $\langle C \bar{C}\rangle \sim \lambda^{-\bar{c}-c+\Delta \bar{f}}$, respectively. One of the terms of each element of the matrix (100) is realized. This depends on which operator are allowed by the symmetry. For example, for the $(1,1)$ element for the Yukawa matrix, interactions $\lambda^{2 \psi+2 f+\phi} \Psi F \Psi F \Phi, \lambda^{2 \psi+f+\bar{c}+2 \phi} \Psi F \Psi \bar{C} \Phi \Phi$ and $\lambda^{2 \psi+2 \bar{c}+3 \phi} \Psi \bar{C} \Phi \Psi \bar{C} \Phi \Phi$, which are allowed if $2 \psi+2 f+\phi \geq 0,2 \psi+f+\bar{c}+2 \phi \geq 0$ and $2 \psi+2 \bar{c}+3 \phi \geq 0$ are satisfied, induce $\lambda^{2 m+2 \Delta f}, \lambda^{2 m+\Delta \bar{f}+\Delta f}$ and $\lambda^{2 m+2 \Delta \bar{f}}$, respectively. That is, the terms that include $\Phi \bar{C}(C \bar{C})$ have $\lambda^{-\delta}$ times larger couplings than the terms that include $\bar{F}(F)$ instead of $\Phi \bar{C}(C \bar{C})$.

In the following arguments, we assume $\Delta \bar{f}=\frac{1}{2}$ and we discuss a possibility that $\lambda^{2 \Delta \bar{f}} \sim 0.22$ becomes the Cabibbo angle ${ }^{7}$. Here we introduce half integer charges and set $c=\phi-\frac{1}{2}$. In order to yield the suitable Cabibbo angle, the difference between the $(2,2)$ element and the $(1,2)$ element should be $2 \Delta \bar{f}$, and thus the $(1,2)$ element is $2 m$ or $2 m-\Delta f+\Delta \bar{f}$, and the $(2,2)$ element is $2 m-2 \Delta \bar{f}$ or $2 m-\Delta \bar{f}-\Delta f$, respectively. If $\phi+\bar{c}>\bar{f}$, when $\Psi \Psi \Phi\left\langle\bar{F}^{2}\right\rangle$ or $\Psi \Psi \Phi\langle\Phi \bar{C} \bar{F}\rangle$ is allowed by the symmetry, then $\Psi \Psi \Phi\left\langle\Phi^{2} \bar{C}^{2}\right\rangle$ is also allowed, which results in $2 m-2 \Delta f$ in the $(2,2)$ element. Therefore, we assume that

$$
\phi+\bar{c}<\bar{f} .
$$

The latter of the $(1,2)$ element comes from $\Psi \Psi \Phi\langle\Phi \bar{C} C \bar{C}\rangle$, which is allowd if $2 m \geq$ $-(\phi+c+2 \bar{c})=-2(\phi+\bar{c})+\frac{1}{2}$. Then, $\Psi \Psi \Phi\langle\Phi C \bar{C} \Phi \bar{C}\rangle$ is also allowed, which results in $2 m-2 \Delta f$ in the $(2,2)$ element. And thus, the Cabibbo angle becomes too small. Therefore, the $(2,2)$ and $(1,2)$ elements must be $2 m-2 \Delta \bar{f}$ and $2 m$, respectively. And if the $(2,3)$ element is $m-\Delta f$, which comes from $\Psi_{3} \Psi \Phi\langle\Phi \bar{C}\rangle$, a charge relation, $m \geq-(\phi+\bar{c})$, must be satisfied, and thus $\Psi \Psi \Phi\langle\Phi \bar{C} \Phi \bar{C}\rangle$ is also allowed, which must be forbidden to reproduce the suitable Cabibbo angle. In summary, the following elements are selected :

$$
\left(\begin{array}{ccc}
\text { "any" } & 2 m & \text { "any" } \\
2 m & 2 m-2 \Delta \bar{f} & m-\Delta \bar{f} \\
\text { "any" } & m-\Delta \bar{f} & 0
\end{array}\right),
$$

where "any" suggests any terms are possible. This condition is expressed in terms of the anomalous $U(1)$ charges as

$$
\phi+\bar{c}+\bar{f}<-2 m
$$

to forbit the term $\Psi \Psi \Phi\langle\Phi \bar{C} \bar{F}\rangle$ which yealds $2 m-\Delta f-\Delta \bar{f}$ for the $(2,2)$ element. Together with the condition (92) and the definition of $k, 3 k=\phi+\bar{c}+f$, we get

$$
\phi<2 k-\frac{2}{3} m .
$$

By the way, when $\phi+\bar{c}+\bar{f}<-2 m, \phi>c$, and Eq. (101) hold, $m$ is smaller than $-(c+\bar{c})$, that is $\Psi_{3} \Psi \Phi\langle\bar{C} C\rangle$ is forbidden by the SUSY-zero mechanism. This means that the $(1,3)$ component of the matrix becomes $m+\Delta f$ that is induced from the interaction $\Psi \Psi_{3} \Phi F$, or the $(1,3)$ element of the corresponding Yukawa matrix vanishes. The important point

\footnotetext{
${ }^{7}$ In order to obtain $r \geq \frac{1}{2}, \Delta f+\Delta \bar{f}>1$ is required if $c-\phi<0$. In that situation, to obtain the suitable value for the Cabibbo mixing, it is a reasonable requirement that either of $\Delta f$ and $\Delta \bar{f}$ becomes $\frac{1}{2}$. Because $\Delta f>\Delta \bar{f}$, we assume that $\Delta \bar{f}=\frac{1}{2}$ here.
} 
Table 8: An example of the charge assignments for the 3rd strategy : Signs denote the $Z_{2}$ symmetry that play the same role as the $Z_{2}$ symmetry introduced in Table 4 . We assume $\langle F \bar{F}\rangle \sim \lambda^{\delta} \lambda^{-(f+\bar{f})}, \delta=4 / 3$ and $\lambda \sim \sin \theta_{C}$. This charge assignment yields $r=11 / 12$ and $l=-2-\delta$. Odd quarter integer charges of the matter fields $\left(\Psi_{3}, \Psi_{a}\right)$ guarantees that the $R$-parity is automatically conserved.

\begin{tabular}{|c|c|c|}
\hline & non-vanishing VEV & vanishing VEV \\
\hline $\mathbf{7 8}$ & $A(a=-1 / 2 ;-)$ & $A^{\prime}\left(a^{\prime}=3 ;-\right)$ \\
$\mathbf{2 7}$ & $\Phi(\phi=-7 / 2 ;+), C(c=-4 ;+)$ & $C^{\prime}\left(c^{\prime}=15 / 2 ;-\right)$ \\
& & $\Psi_{3}\left(\psi_{3}=7 / 4 ;+\right), \Psi_{a}(\psi=17 / 4 ;+)$ \\
$\overline{\mathbf{2 7}}$ & $\bar{C}_{a}(\bar{c}=0 ;+)$ & $\bar{C}^{\prime}\left(\bar{c}^{\prime}=19 / 2 ;-\right)$ \\
$\mathbf{1}$ & $\bar{F}_{a}(\bar{f}=-2 ;+), F_{a}(f=1 ;+)$ & \\
& $\Theta(\theta=-1 / 2 ;+), Z_{i}\left(z_{i}=-1 ;-\right)$ & \\
\hline
\end{tabular}

is that the $(1,3)$ element is larger than the expected value, $(2,3)+(1,2)-(2,2)=m+\Delta \bar{f}$, that is, the $(1,3)$ element of the corresponding Yukawa matrix is smaller than the expected value. Then without the term $\Psi_{3} \Psi C \bar{F}$, the down quark mass and the electron mass become too small (see Appendix A.1 for the detail arguments). Therefore, the term $\Psi_{3} \Psi C \bar{F}$ is required. This requirement is written as

$$
\bar{f} \geq-(m+c-\phi)=-m+\frac{1}{2} .
$$

Together with the above condition (103), we get

$$
\bar{c}<-\phi-m-\frac{1}{2}
$$

Here, we impose one more condition that keep the gauge couplings in the perturbative region. This is realized by $a=-\frac{1}{2}$. Then, in order to allow $A^{\prime} A \Phi \bar{C} F$ to give mass to PNG modes while forbidding $A^{\prime} A^{7}$, we should take $k \geq-5 / 6$. Because smaller $k$ is preferred in order to suppress the FCNC processes, we set the smallest possible value $k=-\frac{5}{6}$.

Because $m$ should be around $\frac{5}{2}-3$ to obtain the hierarchy between the up-type quark masses of the 2nd and 3rd generation, the condition (104) leads to $\phi \lesssim-\frac{10}{3}$, and we set $\phi=-\frac{7}{2}$. If we take $m=\frac{5}{2}, \bar{c} \leq 0$ is led from (106), resulting $f \geq 1$ due to the definition of $k$. Since a larger $f$ leads to a larger $\Delta f$ and therefore a smaller $\epsilon$, which becomes less natural to realize. Thus we take $\bar{c}=0$ and $f=1$. Then, $\delta=\frac{4}{3}$ and $r=\frac{11}{12}$. And the condition $\langle\mathbf{1}\rangle \sim \lambda^{-k} \geq\langle\bar{F}\rangle$ and $\bar{f} \geq-3 \bar{c}-2 f$ make $-4 / 3 \geq \bar{f} \geq-2$, and we fix $\bar{f}=-2$.

Table 8 summarizes the charge assignment. $\left(a^{\prime}, c^{\prime}, z\right)$ are determined as in the previous strategies in 4.2 .1 and 4.2 .2 while $\bar{c}^{\prime}$ is determined to allow $\bar{C}^{\prime} \bar{C} \bar{F} A \Phi \Phi$. This term is required to mix $\mathbf{1 6}_{C}$ component into the massless doublet Higgs, which brings $S U(2)_{R}$ breaking effect into the Yukawa couplings. This $S U(2)_{R}$ breaking effect is needed because in this case $\Psi \Psi \Phi C \bar{C} \bar{F}$ is forbidden.

Note that the interactions which have the total charges larger than $-(c+\phi+2 \bar{c})$ can couple with a singlet operator $\Phi C \bar{C}^{2}$. Because $\lambda^{\phi+c+2 \bar{c}}\left\langle\Phi C \bar{C}^{2}\right\rangle \sim \epsilon^{-1} \gg 1$, the coefficients of such interactions are enhanced by factor $\epsilon^{-1}$, In the Majorana mass matrix and the 
superheavy Higgs mass matrices, such interactions appear and the detail analysis is done in Appendix A. The results are that the parameter $l$ shifts from the naively evaluated value by $-\delta$, and the gauge coupling unification is realized more naturally for the fixed $m_{C}^{\text {eff }}$, which is given as $\lambda^{-\frac{37}{4} 8}$. And the parameter $\delta_{\mathbf{1 0}}$ and $\delta_{\overline{\mathbf{5}}}$ are estimated as

$$
\delta_{10} \sim\left(\begin{array}{ccc}
\lambda^{5 / 3} & \lambda^{8 / 3} & \lambda^{3} \\
\lambda^{8 / 3} & \lambda^{5 / 3} & \lambda^{2} \\
\lambda^{3} & \lambda^{2} & 1
\end{array}\right), \quad \delta_{\overline{\mathbf{5}}} \sim\left(\begin{array}{ccc}
\lambda^{5 / 3} & \lambda^{1+r} & \lambda^{2} \\
\lambda^{1+r} & \lambda & \lambda^{2-r} \\
\lambda^{2} & \lambda^{2-r} & \lambda^{5 / 3}
\end{array}\right) .
$$

These values give larger FCNC processes than those obtained by the 1 st and the 2nd strategy (97). This is because we adopt $a=-\frac{1}{2}$, which is required in order to suppress the divergence of the gauge couplings. The difference from the previous cases is that due to the special charge assignment $\bar{C} C \bar{F}$ cannot appear in the Yukawa interactions, and therefore, the $S U(2)_{R}$ breaking must be realized through the mixings in the MSSM Higgs. This requirement results in the larger $U(1)_{A}$ charge for $\bar{C}^{\prime}$ field, which increases the gauge couplings at the high energy scale.

\section{$4.3 \quad E_{6} \times S U(3)_{H}$}

In this subsection, we consider $E_{6} \times S U(3)_{H}$ model, where three $\Psi$ and three $\overline{\mathbf{2 7}}\left(\bar{C}, \bar{\Phi}, \bar{C}^{\prime}\right)$ are a triplet and an anti-triplet of $S U(3)_{H}$, respectively. In this case, the anomaly of $S U(3)_{H}$ of the matter sector is cancelled by that of the three $\overline{\mathbf{2 7}}$, in contrast to the case of Ref. 15] where some additional fields must be required for the anomaly cancellation.

In order to yield the large top Yukawa coupling, $S U(3)_{H}$ should be broken near the cutoff scale. Suppose that $S U(3)_{H}$ is broken into $S U(2)_{H}$ at the cutoff scale by the VEVs $\langle E\rangle=\langle\bar{E}\rangle \sim \lambda^{-\frac{1}{2}(e+\bar{e})}=1$ and the effective charges can be defined, that is, $e+\bar{e}=0$ is satisfied. Then it can be shown that the effective theory with $S U(2)_{H}$ can be identified with a certain $S U(2)_{H}$ model that have the same $U(1)_{A}$ charges as the effective charges in the effective $S U(2)_{H}$ model. The essential point is that all the interactions in the $S U(2)_{H}$ model can be induced from the interactions in the $S U(3)_{H}$ model. For example, $\lambda^{2 \psi_{3}+\phi} \Psi_{3} \Psi_{3} \Phi$ in $S U(2)_{H}$ model can be obtained from the interaction $\lambda^{2 \psi+2 \bar{e}+\phi} \Psi \bar{E} \Psi \bar{E} \Phi$ by developing the $\mathrm{VEV}\langle\bar{E}\rangle \sim 1$. Note that the coefficient of the effective interaction is determined by the effective charges, that is, $\lambda^{2 \psi+2 \bar{e}+\phi}\langle\bar{E}\rangle^{2} \sim \lambda^{2 \tilde{\psi}_{3}+\phi}$, where $\tilde{\psi}_{3}$ is the effective charge of $\Psi_{3}$ of the effective $S U(2)_{H}$ model. Therefore, it is obvious that the total charge of a interaction in the $S U(3)_{H}$ models nothing but the total effective charge of the corresponding interaction in the effective $S U(2)_{H}$ model because $S U(3)_{H}$ is broken at the cutoff scale. Thus, if a term is forbidden by the SUSY-zero mechanism in the $S U(3)_{H}$ model, the corresponding term in the $S U(2)_{H}$ model is also forbidden by the SUSYzero mechanism. Hence, the effective $S U(2)_{H}$ model can be described by the $S U(2)_{H}$ model. Conversely, if an $S U(2)_{H}$ model is found in which the $U(1)_{A}$ charges are the same as the effective charges of an $S U(3)_{H}$ model, then an $S U(3)_{H}$ model can be found straightforwardly. Note that for $S U(2)_{H}$ models, the arguments in the previous section can be applied, which makes the discussion much simpler.

\footnotetext{
${ }^{8}$ In this case, as shown in Appendix [A] the main mode of $H_{u}$ is included in $\mathbf{1 0}_{\Phi}$ and that of $H_{d}$ is in $\mathbf{1 6}_{C}$. And $m_{C}^{\text {eff }}$ is given by the relation $\lambda^{2 c+\phi}\langle C\rangle$.
} 


\subsection{1 $S U(2)_{H}$ models for $S U(3)_{H}$ models}

In order to extend the horizontal symmetry to $S U(3)_{H}$, the difference $m=\psi-\psi_{3}$ is required to be the same as $\bar{m} \equiv \bar{c}^{\prime}-\bar{c}$. In the 3rd strategy, a large $\bar{c}^{\prime}$ is needed to allow $\bar{C}^{\prime} \bar{C} \bar{F} A \Phi \Phi$, and it is difficult to satisfy the above requirement. Thus we examine the 1st and 2nd strategies in following.

The charge assignments shown in Table 7 still have discrepancy between $m=\psi-\psi_{3}=$ $\frac{5}{2}$ and $\bar{m}=\bar{c}^{\prime}-\bar{c}=\frac{9}{2}$. Note that phenomenologically viable value of $m$ is around $\frac{5}{2}-3$. Thus, models with smaller $\bar{m}$ is needed. Since $\left(f, z_{i}, z_{C}, \bar{c}^{\prime}\right)$ are set as $\left(k+\Delta f, a-\frac{1}{2}, \phi-\right.$ $\left.c-\frac{1}{2},-c-z_{C}-z_{i}\right)$ in the 1st and 2nd strategies, $\bar{m}$ is written as

$$
\bar{m}=\left(\frac{1}{2}-\phi-\left(a-\frac{1}{2}\right)\right)-\bar{c}=2 \times \frac{1}{2}-3 k+f-a=2 \times \frac{1}{2}+\Delta f-2 k-a .
$$

This means that in order to obtain a smaller $\bar{m}$, larger $a$ and $k$ are required. We can construct such models (see Table 9), although the FCNC processes are not suppressed sufficiently:

$$
\delta_{\mathbf{1 0}} \sim\left(\begin{array}{ccc}
\lambda & \lambda^{2} & \lambda^{3} \\
\lambda^{2} & \lambda & \lambda^{2} \\
\lambda^{3} & \lambda^{2} & 1
\end{array}\right), \quad \delta_{\overline{\mathbf{5}}} \sim\left(\begin{array}{ccc}
\lambda & \lambda^{1+r} & \lambda^{2} \\
\lambda^{1+r} & \lambda & \lambda^{2-r} \\
\lambda^{2} & \lambda^{2-r} & \lambda
\end{array}\right) .
$$

Table 9: Examples of the charge assignments of $S U(2)_{H}$ models that can be embedded into $S U(3)_{H}$ models : Signs denote the $Z_{2}$ symmetry that play the same role as the $Z_{2}$ symmetry introduced in Table 4 . When $c \geq \phi$, we impose an additional $Z_{2}$ symmetry and introduce a singlet field $Z_{C}$. These models result in the universal sfermion masses, but the degree of the universality is not sufficient to suppress the FCNC processes.

\begin{tabular}{|c|c|c|}
\hline & non-vanishing VEV & vanishing VEV \\
\hline $\mathbf{7 8}$ & $A(a=-1 / 2 ;-)$ & $A^{\prime}\left(a^{\prime}=3 ;-\right)$ \\
$\mathbf{2 7}$ & $\Phi(\phi=-7 / 2 ;+), C(c=-4,-7 / 2 ;+)$ & $C^{\prime}\left(c^{\prime}=15 / 2 ;-\right)$ \\
& & $\Psi_{3}\left(\psi_{3}=7 / 4 ;+\right), \Psi_{a}(\psi=19 / 4 ;+)$ \\
$\overline{\mathbf{2 7}}$ & $\bar{C}_{a}(\bar{c}=2 ;+)$ & \\
$\mathbf{1}$ & $\bar{C}^{\prime}\left(\bar{c}^{\prime}=5 ;-\right)$ \\
& $\bar{F}_{a}(\bar{f}=-2 ;+), F_{a}(f=0 ;+)$ & \\
& $\Theta(\theta=-1 / 2 ;+), Z_{i}\left(z_{i}=-1 ;-\right)$ & \\
& $Z_{C}\left(z_{C}=-,-1 / 2 ;+\right)$ & \\
\hline
\end{tabular}

In order to improve the suppression of the FCNC processes, we have to change some assumptions. If we employ the other choice of $Z_{2}$ parity introduced in $\$ 4.2 .2$ for $\bar{c}^{\prime}$ as in the footnote there. By this choice, we can set $\bar{c}^{\prime}=-c-z_{i}$, instead of $\bar{c}^{\prime}=-c-z_{C}-z_{i}$, so that $\bar{C}^{\prime}(A+Z) C$ is allowed. This can reduce $\bar{m}$, and we can construct a model that suppresses the FCNC process to the same level as in the models introduced in 4.2 .2 and is able to be embedded into $S U(3)_{H}$ model. (See Table 10) Actually, the parameters $\delta_{\mathbf{1 0}}$ and $\delta_{\overline{5}}$ are the same expression as in the Eqs. (97). 
Table 10: An example of the charge assignments of $S U(2)_{H}$ models that can be embedded into $S U(3)_{H}$ models : Signs denote the $Z_{2}$ symmetry that play the same role as the $Z_{2}$ symmetry introduced in Table 4 . We impose an additional $Z_{2}$ symmetry and introduce a singlet field $Z_{C}$. The FCNC processes are suppressed to the same level as in models in Table 7 This charge assignment yields $r=1$ and $l=-5 / 2$. Odd quarter integer charges of the matter fields $\left(\Psi_{3}, \Psi_{a}\right)$ guarantees that the $R$-parity is automatically conserved.

\begin{tabular}{|c|c|c|}
\hline & non-vanishing VEV & vanishing VEV \\
\hline $\mathbf{7 8}$ & $A(a=-1 ;-)$ & $A^{\prime}\left(a^{\prime}=5 ;-\right)$ \\
$\mathbf{2 7}$ & $\Phi(\phi=-7 / 2 ;+), C(c=-5 / 2 ;+)$ & $C^{\prime}\left(c^{\prime}=8 ;-\right)$ \\
& & $\Psi_{3}\left(\psi_{3}=7 / 4 ;+\right), \Psi_{a}(\psi=19 / 4 ;+)$ \\
$\overline{\mathbf{2 7}}$ & $\bar{C}_{a}(\bar{c}=1 ;+)$ & $\bar{C}^{\prime}\left(\bar{c}^{\prime}=4 ;-\right)$ \\
$\mathbf{1}$ & $\bar{F}_{a}(\bar{f}=-2 ;+), F_{a}(f=-1 / 2 ;+)$ & \\
& $\Theta(\theta=-1 / 2 ;+), Z_{i}\left(z_{i}=-3 / 2 ;-\right)$ & \\
& $Z_{C}\left(z_{C}=-3 / 2 ;+\right)$ & \\
\hline
\end{tabular}

\subsection{2 $S U(3)_{H}$ models}

Now, we treat $S U(3)_{H}$ models. The Higgs content is summarized in Table 11]. Each

Table 11: The Higgs content of $E_{6} \times S U(3)_{H} \times U(1)_{A}$ models expect for singlets: Here $S U(3)_{H}$ triplets and anti-triplets are denoted by the lower and upper index $\alpha$, respectively. All the non-vanishing VEVs are shown, and their magnitudes are formally written by introducing parameters $\Delta \phi$ etc.. One or more discreate symmetries are introduced according to need.

\begin{tabular}{|c|c|c|}
\hline & non-vanishing VEV & vanishing VEV \\
\hline $\mathbf{7 8}$ & $A\left(\left\langle\mathbf{4 5}_{A}\right\rangle \sim \lambda^{-a}\right)$ & $A^{\prime}$ \\
$\mathbf{2 7}$ & $\Phi\left(\left\langle\mathbf{1}_{\Phi}\right\rangle \sim \lambda^{-(\phi-\Delta \phi)}\right), C\left(\left\langle\mathbf{1}_{C}\right\rangle \sim \lambda^{-(c-\Delta c)}\right)$ & $C^{\prime}$ \\
$\overline{\mathbf{2 7}}$ & $\bar{C}^{\alpha}\left(\left\langle\overline{\mathbf{1 6}}_{\bar{C}_{1}}\right\rangle \sim \lambda^{-(\bar{c}+\Delta c-\Delta f-\Delta e / 2)},\left\langle\overline{\mathbf{1}}_{\bar{C}_{2}}\right\rangle \sim \lambda^{-(\bar{c}+\Delta \phi+\Delta f-\Delta e / 2)}\right)$ & \\
$\mathbf{1}$ & $F_{\alpha}\left(\left\langle F_{2}\right\rangle \sim \lambda^{-(f-\Delta f+\Delta e / 2)}\right), \bar{F}^{\alpha}\left(\left\langle\bar{F}_{2}\right\rangle \sim \lambda^{-(\bar{f}+\Delta f-\Delta e / 2)}\right)$ & \\
& $E_{\alpha}\left(\left\langle E_{3}\right\rangle \sim \lambda^{-(e-\Delta e)}\right), \bar{E}^{\alpha}\left(\left\langle\bar{E}_{3}\right\rangle \sim \lambda^{-(\bar{e}+\Delta e)}\right)$ & \\
\hline
\end{tabular}

component of a triplet $\Psi_{\alpha}$ and an anti-triplet $\bar{C}^{\alpha}$ can be picked up as

$$
\begin{aligned}
\left(\Psi_{1}, \Psi_{2}, \Psi_{3}\right) & \sim(\Psi E F, \Psi \bar{F}, \Psi E) \\
\left(\bar{C}_{1}, \bar{C}_{2}, \bar{C}_{3}\right) & \sim(\bar{C} \bar{E} \bar{F}, \bar{C} F, \bar{C} E),
\end{aligned}
$$

and the effective charge of each element is given as

$$
\begin{aligned}
\widetilde{\psi} & =(\psi+\Delta f+\Delta e / 2, \psi-\Delta f+\Delta e / 2, \psi-\Delta e) \\
\widetilde{\bar{c}} & =(\bar{c}-\Delta f-\Delta e / 2, \bar{c}+\Delta f-\Delta e / 2, \bar{c}+\Delta e) .
\end{aligned}
$$

\footnotetext{
${ }^{9}$ Note that the $S U(3)_{H}$ horizontal symmetry in this model is anomaly free.
} 
This means that, providing $e=-\bar{e}=\Delta e$ and integrating out $E$ and $\bar{E}$, we get a $S U(2)_{H}$ model where $\left(\psi, \psi_{3}, \bar{c}, \bar{c}^{\prime}, \bar{f}, f\right)$ are given as $\left(\psi+e / 2, \psi_{3}-e, \bar{c}-e / 2, \bar{c}^{\prime}+e, \bar{f}-e / 2, f+e / 2\right)$ in terms of the charges in the $S U(3)_{H}$ model $^{10}$. Conversely, we can construct an $S U(3)_{H}$ model with $e=-e=2$ as shown in Table 12 from a $S U(2)_{H}$ model in Table 10. Here,

Table 12: An example of the charge assignments of $S U(3)_{H}$ models : Signs denote the $Z_{2}$ symmetry that play the same role as the $Z_{2}$ symmetry introduced in Table 4 . We impose an additional $Z_{2}$ symmetry and introduce a singlet field $Z_{C}$. The FCNC processes are suppressed to the same level as in models in Table [7. This charge assignment yields $r=1$ and $l=-5 / 2$. Odd quarter integer charge of the matter field $\left(\Psi_{\alpha}\right)$ guarantees that the $R$-parity is automatically conserved.

\begin{tabular}{|c|c|c|}
\hline & non-vanishing VEV & vanishing VEV \\
\hline $\mathbf{7 8}$ & $A(a=-1 ;-)$ & $A^{\prime}\left(a^{\prime}=5 ;-\right)$ \\
$\mathbf{2 7}$ & $\Phi(\phi=-7 / 2 ;+), C(c=-5 / 2 ;+)$ & $C^{\prime}\left(c^{\prime}=8 ;-\right)$ \\
& & $\Psi_{\alpha}(\psi=15 / 4 ;-)$ \\
$\overline{\mathbf{2 7}}$ & $\bar{C}^{\alpha}(\bar{c}=2 ;+)$ & \\
$\mathbf{1}$ & $F_{\alpha}(f=-3 / 2 ;+), \bar{F}^{\alpha}(\bar{f}=-1 ;-)$ & \\
& $E_{\alpha}(e=2 ;-), \bar{E}^{\alpha}(\bar{e}=-2 ;-)$ & \\
& $\Theta(\theta=-1 / 2 ;+), Z_{i}\left(z_{i}=-3 / 2 ;-\right)$ & \\
& $Z_{C}\left(z_{C}=-3 / 2 ;+\right)$ & \\
\hline
\end{tabular}

parity assignment of the additional $Z_{2}$ symmetry for (anti)triplet fields $(\Psi, \bar{C}, F, \bar{F}, E, \bar{E})$ is $(-,+,+,-,-,-)$, so that $\bar{C}_{a}(a=1,2)$ and $\Psi_{\alpha}(\alpha=1,2,3)$ have even parity while $\bar{C}_{3}$ has odd parity, and the others have the same parity as in the $S U(2)_{H}$ model. This parity plays essentially the same role as that in the $S U(2)_{H}$ model in Table 10.

\section{$5 \quad$ Summary and Discussion}

In this paper, we have investigated $S O(10)$ or $E_{6}$ SUSY-GUTs with an anomalous $U(1)$ symmetry and an $S U(2)_{H}$ or $S U(3)_{H}$ horizontal symmetry, where some of GUT-breaking Higgs belong to non-trivial representations of the horizontal symmetry. We have found it possible to unify the Higgs sectors for the GUT symmetry and the horizontal symmetry. It is interesting that for $S U(3)_{H}$ models, $S U(3)_{H}$ gauge anomaly is cancelled between the triplet matter $\Psi_{a}$ and the anti-triplet Higgs $\bar{C}^{a}$.

Unfortunately, the unification of the Higgs sectors of the GUT symmetry and the horizontal symmetry results in too large FCNC processes. This is because in the scenario of the GUT with the anomalous $U(1)$ symmetry the cutoff scale, $\Lambda$, must be around the usual GUT scale, $2 \times 10^{16} \mathrm{GeV}$, to realize the natural gauge coupling unification [18] and the true GUT scale in our scenario is difficult to be smaller than $\lambda^{2} \Lambda$, which is the

\footnotetext{
${ }^{10} \mathrm{As}$ for the $Z_{2}$-parities, we can find those of each component from Eqs. (110) and (111). In addition, for example, $\bar{C} \bar{E} \bar{C} \Phi$ and $\bar{C} \bar{E} \bar{F} Z_{C}$ (whose charges are usually smaller than that of $\bar{C} \bar{E} \bar{F}$ ) pick up $C_{1}$ component with another parity assignments (they may be same as the previous one).
} 
sufficient value for suppressing the FCNC processes. At present, we do not know the meaning of this fact. This fact may mean that another mechanism is required to realize the universality of sfermion masses, or that the fields in the Higgs sector of the GUT symmetry do not have non-trivial quantum numbers under the horizontal symmetry, or that the anomalous $U(1)_{A}$ is given up although the GUT models with the anomalous $U(1)_{A}$ solve the doublet-triplet splitting problem and realize the natural gauge coupling unification with generic interactions. However, we hope that the arguments in this paper give a hint in finding the real grand unified theory, which we expect to be just around the corner.

\section{Acknowledgement}

This work is supported by a Grant-in-Aid for the 21st Century COE" Center for Diversity and Universality in Physics". N.M. is supported in part by Grants-in-Aid for Scientific Research from the Ministry of Education, Culture, Sports, Science and Technology of Japan.

\section{A Effects of deviation from effective charge}

In this appendix, we analyse the model defined in Table 8 , in which the VEV relations (9) are not satisfied, that is, the effective charges are not well defined.

\section{A.1 $5-\overline{5}$ mass matrix of matters}

First, we consider the mass matrix of $\mathbf{5 - \overline { 5 }}$ components of $\Psi_{a}$ and $\Psi_{3}$. Because of the decomposition of the fundamental representation of $E_{6}$ (8) , this matrix becomes $3 \times 6$. The mass terms of $(\mathbf{1 0}, \mathbf{5})-(\mathbf{1 0}, \overline{\mathbf{5}})$ are given from $\Psi \Psi\langle\Phi\rangle$ and those of $(\mathbf{1 0}, \mathbf{5})-(\mathbf{1 6}, \overline{\mathbf{5}})$ are given from $\Psi \Psi\langle C\rangle$. For the charge assignment in Table 8 , this matrix is given in terms of the exponent as

$$
\begin{aligned}
& \Psi_{1}(\mathbf{1 0}, \overline{\mathbf{5}}) \quad \Psi_{2}(\mathbf{1 0}, \overline{\mathbf{5}}) \quad \Psi_{3}(\mathbf{1 0}, \overline{\mathbf{5}}) \quad \Psi_{1}(\mathbf{1 6}, \overline{\mathbf{5}}) \quad \Psi_{2}(\mathbf{1 6}, \overline{\mathbf{5}}) \quad \Psi_{3}(\mathbf{1 6}, \overline{\mathbf{5}}) \\
& \begin{array}{l}
\Psi_{1}(\mathbf{1 0}, \mathbf{5}) \\
\Psi_{2}(\mathbf{1 0}, \mathbf{5}) \\
\Psi_{3}(\mathbf{1 0}, \mathbf{5})
\end{array}\left(\begin{array}{cccccc}
6+\delta & 5 & 3+\delta & 6+\delta+r & 5+r & 3+\delta+r \\
5 & 4 & 2 & 5+r & 4+r & 2+r \\
3+\delta & 2 & 0 & 3+\delta+r & 2+r & -
\end{array}\right)-k,
\end{aligned}
$$

where "-" means that the corresponding element of the $3 \times 6$ mass matrix is forbidden

by the SUSY-zero mechanism. From this matrix, we can find the three $\overline{\mathbf{5}}$ that remain massless are given as

$$
\begin{aligned}
& \overline{\mathbf{5}}_{\mathbf{1}}=\Psi_{1}(\mathbf{1 6}, \overline{\mathbf{5}})+\lambda^{3} \Psi_{3}(\mathbf{1 6}, \overline{\mathbf{5}})+\lambda^{3+\delta+r} \Psi_{3}(\mathbf{1 0}, \overline{\mathbf{5}})+\lambda^{1+\delta+r} \Psi_{2}(\mathbf{1 0}, \overline{\mathbf{5}}), \\
& \overline{\mathbf{5}}_{\mathbf{2}}=\Psi_{1}(\mathbf{1 0}, \overline{\mathbf{5}})+\lambda^{3-r} \Psi_{3}(\mathbf{1 6}, \overline{\mathbf{5}})+\lambda^{3+\delta} \Psi_{3}(\mathbf{1 0}, \overline{\mathbf{5}})+\lambda^{1+\delta} \Psi_{2}(\mathbf{1 0}, \overline{\mathbf{5}}), \\
& \overline{\mathbf{5}}_{\mathbf{3}}=\Psi_{2}(\mathbf{1 6}, \overline{\mathbf{5}})+\lambda^{2} \Psi_{3}(\mathbf{1 6}, \overline{\mathbf{5}})+\lambda^{2+r} \Psi_{3}(\mathbf{1 0}, \overline{\mathbf{5}})+\lambda^{r} \Psi_{2}(\mathbf{1 0}, \overline{\mathbf{5}}) .
\end{aligned}
$$


In order to obtain the Yukawa couplings of quarks and leptons, we must know the origin of the MSSM Higgs $H_{u}$ and $H_{d}$. Here we write just the results:

$$
H_{u} \sim \mathbf{1 0} 0_{\Phi}+\lambda^{\frac{1}{2}} \mathbf{1 0} 0_{C}, \quad H_{d} \sim \mathbf{1 6} \mathbf{6}_{C}+\lambda^{c-\phi+r} \mathbf{1 0}_{\Phi}+\lambda^{r} \mathbf{1 0} \mathbf{0}_{C}
$$

which will be shown in Appendix A.3. Then we obtain the following Yukawa matrix and mixing matrix for the $\overline{\mathbf{5}}$ sector as

$$
y_{\overline{\mathbf{5}}}=\left(\begin{array}{ccc}
\lambda^{6+\delta} & \lambda^{5} & \lambda^{3} \\
\lambda^{6+\delta-r} & \lambda^{5-r} & \lambda^{3-r} \\
\lambda^{5} & \lambda^{4} & \lambda^{2}
\end{array}\right) \lambda^{r-\frac{1}{2}}, \quad V_{\overline{\mathbf{5}}}=\left(\begin{array}{ccc}
1 & \lambda^{r} & \lambda \\
\lambda^{r} & 1 & \lambda^{1-r} \\
\lambda & \lambda^{1-r} & 1
\end{array}\right)
$$

Note that if $\Psi_{3} \Psi C \bar{F}$ is not allowed, the $(2,6)$ and $(3,5)$ elements of the matrix (114) become "-". Then the light three $\overline{\mathbf{5}}$ modes become

$$
\begin{aligned}
& \overline{\mathbf{5}}_{\mathbf{1}}=\Psi_{1}(\mathbf{1 6}, \overline{\mathbf{5}})+\lambda^{3-\delta} \Psi_{3}(\mathbf{1 6}, \overline{\mathbf{5}})+\lambda^{3+r} \Psi_{3}(\mathbf{1 0}, \overline{\mathbf{5}})+\lambda^{1+r} \Psi_{2}(\mathbf{1 0}, \overline{\mathbf{5}}), \\
& \overline{\mathbf{5}}_{\mathbf{2}}=\Psi_{1}(\mathbf{1 0}, \overline{\mathbf{5}})+\lambda^{3-\delta-r} \Psi_{3}(\mathbf{1 6}, \overline{\mathbf{5}})+\lambda^{3} \Psi_{3}(\mathbf{1 0}, \overline{\mathbf{5}})+\lambda^{1} \Psi_{2}(\mathbf{1 0}, \overline{\mathbf{5}}), \\
& \overline{\mathbf{5}}_{\mathbf{3}}=\Psi_{2}(\mathbf{1 6}, \overline{\mathbf{5}})+\lambda^{2-\delta} \Psi_{3}(\mathbf{1 6}, \overline{\mathbf{5}})+\lambda^{2+r} \Psi_{3}(\mathbf{1 0}, \overline{\mathbf{5}})+\lambda^{r} \Psi_{2}(\mathbf{1 0}, \overline{\mathbf{5}})
\end{aligned}
$$

which leads the Yukawa matrix to

$$
y_{\overline{\mathbf{5}}}=\left(\begin{array}{ccc}
\lambda^{6+\delta} & \lambda^{5} & \lambda^{3} \\
\lambda^{6+\delta-r} & \lambda^{5-r} & \lambda^{3-r} \\
\lambda^{5+\delta} & \lambda^{4} & \lambda^{2}
\end{array}\right) \lambda^{r-\frac{1}{2}-\delta}
$$

yielding the ratio between the 1 st and 3rd eigenvalues as $m_{\overline{\mathbf{5}}_{1}} / m_{\overline{\mathbf{5}}_{\mathbf{3}}} \sim \lambda^{4+\delta}$ which is a bit too small because $\delta=\frac{4}{3}$. Thus, the term $\Psi_{3} \Psi C \bar{F}$ is required.

\section{A.2 Neutrino mass}

Next, we consider the neutrino mass matrix. Their Yukawa couplings are give by $3 \times 6$ matrix because there are two right-handed neutrinos in each $\mathbf{2 7}$. They are given from $\Psi(\mathbf{1 6}, \overline{\mathbf{5}}) \Psi(\mathbf{1 6}, \mathbf{1}) \Phi(\mathbf{1 0}, \mathbf{5})$ and $\Psi(\mathbf{1 0}, \overline{\mathbf{5}}) \Psi(\mathbf{1}, \mathbf{1}) \Phi(\mathbf{1 0}, \mathbf{5})$. The Yukawa matrix is given as

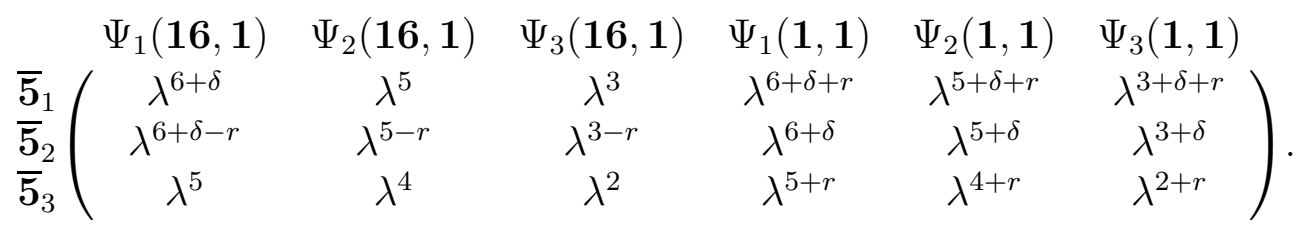

Note that this is written as

$$
\left(\begin{array}{ll}
\lambda^{2} & \lambda^{2+r}
\end{array}\right) \otimes\left(\begin{array}{ccc}
\lambda^{4+\delta} & \lambda^{3} & \lambda^{1} \\
\lambda^{4+\delta-r} & \lambda^{3-r} & \lambda^{1-r} \\
\lambda^{3} & \lambda^{2} & 1
\end{array}\right)
$$

except for the $(1,5),(1,6),(2,5)$ and $(2,6)$ elements, which are smaller than the corresponding elements of the expression (125). 
The Majorana mass matrix of the right-handed neutrinos, $M_{R}$, is a $6 \times 6$ matrix and their elements are given from $\Psi \Psi\langle\bar{C} \bar{C}\rangle$. This matrix is written in terms of the exponent as

$$
\begin{aligned}
& \Psi_{1}(\mathbf{1 6}, \mathbf{1}) \quad \Psi_{2}(\mathbf{1 6}, \mathbf{1}) \quad \Psi_{3}(\mathbf{1 6}, \mathbf{1}) \quad \Psi_{1}(\mathbf{1}, \mathbf{1}) \quad \Psi_{2}(\mathbf{1}, \mathbf{1}) \quad \Psi_{3}(\mathbf{1}, \mathbf{1}) \\
& \Psi_{1}(\mathbf{1 6}, \mathbf{1})\left(\begin{array}{cccccc}
6-2 r-\delta & 5-2 r-2 \delta & 3-2 r-\delta & 6-r-\delta & 5-r-2 \delta & 3-r-\delta
\end{array}\right. \\
& \Psi_{2}(\mathbf{1 6}, \mathbf{1}) \quad \begin{array}{llllll}
5-2 r-2 \delta & 4-2 r & 2-2 r & 5-r-2 \delta & 4-r-3 \delta & 2-r-2 \delta
\end{array} \\
& \Psi_{3}(\mathbf{1 6}, \mathbf{1}) \quad \begin{array}{llllll}
3-2 r-\delta & 2-2 r & - & 3-r-\delta & 2-r-2 \delta & -r-\delta
\end{array} \\
& \begin{array}{lllllll}
\Psi_{1}(\mathbf{1}, \mathbf{1}) & 6-r-\delta & 5-r-2 \delta & 3-r-\delta & 6 & 5-2 \delta & 3
\end{array}
\end{aligned}
$$

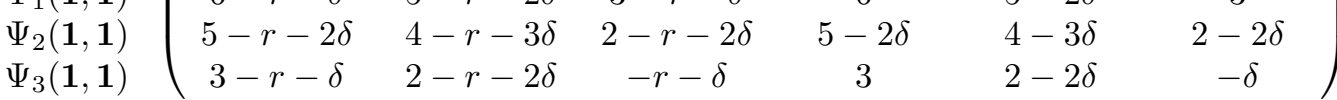

$$
\begin{aligned}
& +3.5-2 \Delta \phi
\end{aligned}
$$

and can be expressed as

$$
\lambda^{3.5-2 \Delta \phi-\delta}\left(\begin{array}{cc}
\lambda^{-2 r} & \lambda^{-r} \\
\lambda^{-r} & 1
\end{array}\right) \otimes\left(\begin{array}{ccc}
\lambda^{6} & \lambda^{5-\delta} & \lambda^{3} \\
\lambda^{5-\delta} & \lambda^{4-2 \delta} & \lambda^{2-\delta} \\
\lambda^{3} & \lambda^{2-\delta} & 1
\end{array}\right)
$$

except for the $(2,2),(2,3),(3,2),(3,3),(4,4),(4,6)$ and $(6,4)$ elements, which are smaller than the corresponding elements of the expression (127). These small elements have only sub-leading contributions to the inverse matrix $M_{R}^{-1}$. Also, the small elements in the expression (124) have only sub-leading contributions to the light neutrino mass. Thus, the correct order of magnitudes of the light neutrino mass are obtained by a calculation using the expressions (125) and (127) instead of (124) and (126), which leads to

$$
\begin{gathered}
\lambda^{-3.5+2 \Delta \phi+\delta}\left\langle H_{u}\right\rangle^{2}\left(\begin{array}{cc}
\lambda^{2} & \lambda^{2+r}
\end{array}\right)\left(\begin{array}{cc}
\lambda^{2 r} & \lambda^{r} \\
\lambda^{r} & 1
\end{array}\right)\left(\begin{array}{c}
\lambda^{2} \\
\lambda^{2+r}
\end{array}\right) \otimes \\
\left(\begin{array}{ccc}
\lambda^{4+\delta} & \lambda^{3} & \lambda^{1} \\
\lambda^{4+\delta-r} & \lambda^{3-r} & \lambda^{1-r} \\
\lambda^{3} & \lambda^{2} & 1
\end{array}\right)\left(\begin{array}{ccc}
\lambda^{-6} & \lambda^{-5+\delta} & \lambda^{-3} \\
\lambda^{-5+\delta} & \lambda^{-4+2 \delta} & \lambda^{-2+\delta} \\
\lambda^{-3} & \lambda^{-2+\delta} & 1
\end{array}\right)\left(\begin{array}{ccc}
\lambda^{4+\delta} & \lambda^{4+\delta-r} & \lambda^{3} \\
\lambda^{3} & \lambda^{3-r} & \lambda^{2} \\
\lambda^{1} & \lambda^{1-r} & 1
\end{array}\right) \\
=\lambda^{-3.5+2 \Delta \phi+\delta} \lambda^{4+2 r}\left(\begin{array}{ccc}
\lambda^{2} & \lambda^{2-r} & \lambda^{1} \\
\lambda^{2-r} & \lambda^{2-2 r} & \lambda^{1-r} \\
\lambda^{1} & \lambda^{1-r} & 1
\end{array}\right)\left\langle H_{u}\right\rangle^{2} .
\end{gathered}
$$

In this case, the parameter $l$ is given as

$$
-(l+5)=-3.5+2 \Delta \phi+\delta+4+2 r .
$$

This is different from the previous expression for $l$ (90) by $\delta$. Note that the determinant of the matrix (128) is smaller than the naively expected determinant of the matrix (129) by a factor $\lambda^{2 \delta}$. This means a cancellation must occur in caluculating the eigenvalues of the matirx (129), and the mass of the lightest neutrino become,

$$
\frac{m_{\nu_{1}}}{m_{\nu_{3}}} \sim \lambda^{2+2 \delta}
$$

which is smaller than the naively expected value from the matrix (129), $\frac{m_{\nu_{1}}}{m_{\nu_{3}}} \sim \lambda^{2}$. 


\section{A.3 Mass matrix of GUT-breaking Higgs}

Finally we examine mass matrices of Higgs that break the $E_{6}$ gauge symmetry. Because the VEV relations (91) are not satisfied in this model, the success of the gauge coupling unification may be spoiled. Actually, the coefficients of some effective interactions are dependent on the original interactions given at the cutoff scale as discusses in 4.2 .3 . that is, the effective charges cannot be defined well. Moreover, if the total charge of a interaction is large enough for the singlet operator, $\bar{C} \bar{C} \Phi C$, to couple with the interaction, the coefficient for the interaction is enhanced by a factor $\lambda^{2 \bar{c}+\phi+c}\langle\bar{C} \bar{C} \Phi C\rangle \sim \epsilon^{-1}$. Such effects, in principle, disturb the gauge coupling unification, which are guaranteed if the effective charges can be well-defined 18. Let us illustrate these by calculating the mass matrix of the $\mathbf{5 - \mathbf { 5 }}$ components of Higgs explicitly as an example. It is given as following $11 \times 11$ matrix.

\begin{tabular}{|c|c|c|c|c|c|c|c|c|c|c|c|}
\hline $\bar{I} \backslash I$ & $10_{C}$ & $10_{\Phi}$ & $16_{C}$ & $16_{\Phi}$ & $16_{A}$ & $16_{A^{\prime}}$ & $10_{\bar{C}_{2}}$ & $10_{\bar{C}_{1}}$ & $16_{C^{\prime}}$ & $10_{C^{\prime}}$ & $10_{\bar{C}^{\prime}}$ \\
\hline $10_{C}$ & 0 & 0 & 0 & 0 & 0 & 0 & 0 & 0 & 0 & 0 & $\lambda^{\frac{11}{2}}$ \\
\hline $10_{\Phi}$ & 0 & 0 & 0 & 0 & 0 & 0 & 0 & 0 & 0 & $\alpha_{I} \lambda^{\frac{4}{3}}$ & $\lambda^{6}$ \\
\hline$\overline{\mathbf{1 6}}_{\bar{C}_{2}}$ & 0 & 0 & 0 & 0 & 0 & 0 & 0 & 0 & $\frac{1}{\epsilon} \lambda^{7}$ & 0 & $\frac{1}{\epsilon^{2}} \lambda^{\frac{43}{4}}$ \\
\hline $10_{\bar{C}_{2}}$ & 0 & 0 & 0 & 0 & 0 & 0 & 0 & $\lambda^{4}$ & $\frac{1}{\epsilon} \lambda^{\frac{95}{12}}$ & $\frac{1}{\epsilon} \lambda^{7}$ & $\frac{1}{\epsilon^{2}} \lambda^{\frac{35}{3}}$ \\
\hline$\overline{\mathbf{1 6}}_{\bar{C}_{1}}$ & 0 & 0 & 0 & 0 & 0 & $\lambda^{\frac{13}{6}}$ & 0 & $\lambda^{\frac{65}{12}}$ & $\frac{1}{\epsilon} \lambda^{\frac{28}{3}}$ & $\frac{1}{\epsilon} \lambda^{\frac{101}{12}}$ & $\frac{1}{\epsilon^{2}} \lambda^{\frac{157}{12}}$ \\
\hline$\overline{\mathbf{1 6}}_{A}$ & 0 & 0 & 0 & 0 & 0 & $\lambda^{\frac{5}{2}}$ & 0 & 0 & $\frac{1}{\epsilon} \lambda^{\frac{29}{3}}$ & $\frac{1}{\epsilon} \lambda^{\frac{35}{4}}$ & $\frac{1}{\epsilon^{2}} \lambda^{\frac{161}{12}}$ \\
\hline $10_{\bar{C}_{1}}$ & 0 & 0 & 0 & 0 & 0 & 0 & $\lambda^{4}$ & $\lambda^{\frac{19}{3}}$ & $\frac{1}{\epsilon} \lambda^{\frac{41}{4}}$ & $\frac{1}{\epsilon} \lambda^{\frac{28}{3}}$ & $\frac{1}{\epsilon^{2}} \lambda^{14}$ \\
\hline$\overline{\mathbf{1 6}}_{A^{\prime}}$ & 0 & 0 & 0 & $\lambda^{\frac{13}{6}}$ & $\lambda^{\frac{5}{2}}$ & $\lambda^{6}$ & 0 & 0 & $\begin{array}{l}\frac{1}{\epsilon} \lambda^{\frac{79}{6}} \\
\end{array}$ & $\frac{1}{\epsilon} \lambda^{\frac{49}{4}}$ & $\frac{\epsilon^{2}}{\frac{1}{\epsilon^{2}} \lambda^{\frac{203}{12}}}$ \\
\hline $10_{C^{\prime}}$ & 0 & $\alpha_{I} \lambda^{\frac{4}{3}}$ & 0 & 0 & 0 & $\lambda^{\frac{73}{12}}$ & $\frac{1}{\epsilon} \lambda^{7}$ & $\frac{1}{\epsilon} \lambda^{\frac{28}{3}}$ & $\frac{1}{\epsilon} \lambda^{\frac{53}{4}}$ & $\frac{1}{\epsilon} \lambda^{\frac{37}{3}}$ & $\frac{1}{\epsilon^{2}} \lambda^{17}$ \\
\hline$\overline{\mathbf{1 6}}_{\bar{C}^{\prime}}$ & 0 & $\lambda^{\frac{61}{12}}$ & $\lambda^{\frac{11}{2}}$ & $\lambda^{6}$ & $\lambda^{\frac{19}{3}}$ & $\lambda^{\frac{59}{6}}$ & $\frac{1}{\epsilon^{2}} \lambda^{\frac{43}{4}}$ & $\frac{1}{\epsilon^{2}} \lambda^{\frac{157}{12}}$ & $\begin{array}{l}\frac{1}{\epsilon^{2}} \lambda^{17} \\
\text {. }\end{array}$ & $\begin{array}{l}\frac{1}{\epsilon^{2}} \lambda^{\frac{193}{12}} \\
\text {. }\end{array}$ & $\frac{\epsilon^{2}}{\frac{1}{\epsilon^{3}} \lambda^{\frac{83}{4}}}$ \\
\hline $10_{\bar{C}^{\prime}}$ & $\lambda^{11 / 2}$ & $\lambda^{6}$ & $\lambda^{\frac{77}{12}}$ & $\lambda^{\frac{83}{12}}$ & $\lambda^{\frac{29}{4}}$ & $\lambda^{\frac{43}{4}}$ & $\frac{1}{\epsilon^{2}} \lambda^{\frac{35}{3}}$ & $\frac{1}{\epsilon^{2}} \lambda^{14}$ & $\frac{1}{\epsilon^{2}} \lambda^{\frac{215}{12}}$ & $\frac{1}{\epsilon^{2}} \lambda^{17}$ & \\
\hline
\end{tabular}

Here, $\alpha_{I}=0$ for the doublet component, which yields additional massless mode only for doublet written as

$$
H_{u} \sim 10_{\Phi}+\lambda^{\phi-c} \mathbf{1 0}_{C}, \quad H_{d} \sim 1 \mathbf{6}_{C}+\lambda^{c-\phi+r} \mathbf{1 0}_{\Phi}+\lambda^{r} \mathbf{1 0} 0_{C}
$$

which are identified with the MSSM doublet Higgs.

In principle, because the VEV realtions are not satisfied, the gauge coupling unification is not guranteed in this model. Of course, the discrepancy is parametrized by $\epsilon$, because if $\epsilon \sim \mathcal{O}(1)$, the natural gauge coupling unification is realized as discussed in Refs. [18]. In fact, the mass spectrum, which is important in estimating the discrepancy, are given as $m_{T}=\lambda^{\frac{11}{2}}, \lambda^{\frac{4}{3}}, \frac{1}{\epsilon} \lambda^{7}, \lambda^{4}, \lambda^{\frac{13}{6}}, 0, \lambda^{4}, \lambda^{\frac{13}{6}}, \lambda^{\frac{4}{3}}, \lambda^{\frac{11}{2}}, \lambda^{\frac{11}{2}}$ for the triplet components and $m_{D}=$ $\lambda^{\frac{11}{2}}, 0, \frac{1}{\epsilon} \lambda^{7}, \lambda^{4}, \lambda^{\frac{13}{6}}, 0, \lambda^{4}, \lambda^{\frac{13}{6}}, \frac{1}{\epsilon} \lambda^{\frac{37}{3}}, \lambda^{\frac{61}{12}}, \lambda^{\frac{11}{2}}$ for the doublet components. Note that $1 / \epsilon$ appear in relatively small elements. This is mainly because the enhancement factor, $\lambda^{2 \bar{c}+\phi+c}\langle\bar{C} \bar{C} \Phi C\rangle \sim \epsilon^{-1}$, can appear in the terms with sufficiently large anomalous $U(1)_{A}$ charges. The ratio det $m_{D} / \operatorname{det} m_{T}$, which is important in estimating the discrepancy, is enhanced by a factor $\epsilon^{-1}$ in this model. This enhancement improves the gauge coupling 
unification because relatively light colored Higgs mass (and therefore, heavy doublet Higgs mass) is prefered. This feaure that the ratio is enhanced and the gauge coupling unification is improved, is comparatively general in this scenario. The reasons are as follows. The typical mass matrix for a $S U(5)$ irreducible representation fields, $X$ and $X^{\prime}\left(\bar{X}\right.$ and $\bar{X}^{\prime}$ are conjugate representation fields) is given as

$$
\left(\begin{array}{cc}
0 & \lambda^{x+\bar{x}^{\prime}} \alpha \\
\lambda^{\bar{x}+x^{\prime}} \alpha & \lambda^{\bar{x}^{\prime}+x^{\prime}}
\end{array}\right),
$$

where the effective charges $x$ and $\bar{x}$ are negative and $x^{\prime}$ and $\bar{x}^{\prime}$ are positive. The $\mathcal{O}(1)$ paramter $\alpha$ is vanishing for one of the component fields for $X, \bar{X}, X^{\prime}$, and $\bar{X}^{\prime}$. ( For $\overline{5}$ and 5 fields, $\alpha=0$ for the doublet Higgs because of the doublet-triplet splitting. In our scenario, such vanishing parameters like $\alpha$ also appear for the Nambu-Goldstone (NG) modes in breaking $E_{6} \rightarrow G_{\mathrm{SM}}$.) Bacause $x$ and $\bar{x}$ are negative and $x^{\prime}$ and $\bar{x}^{\prime}$ are positive, the $(1,1)$ element is vanishing by the SUSY zero mechanism and the non-diagonal elements are larger than the other diagonal $(2,2)$ element. Therefore, when $\alpha \neq 0$, the determinant of the mass matrix is given by the product of the non-diagonal elements, $\lambda^{x+\bar{x}+x^{\prime}+\bar{x}^{\prime}}$. However, when $\alpha=0$, the mass eigenvalues become 0 and $\lambda^{x^{\prime}+\bar{x}^{\prime}}$, which are the smallest non-vanishing eigenvalue among the mass spectrum of $X, X^{\prime}, \bar{X}$, and $\bar{X}^{\prime}$. As discussed in the above, it is plausible that the factor $\epsilon^{-1}$ appears in the $(2,2)$ element, which is enhanced if $\epsilon \ll 1$, that is, the eigenvalue for the component field with the vanishing $\alpha$ is enhanced. For the mass matrix of $\overline{\mathbf{5}}$ and $\mathbf{5}$ fields, if doublet-triplet splitting is realized by a certain mechanism, the mass eigenvalues of doublet components tend to be enhanced when $\epsilon \ll 1$. (The effect of the NG modes can be neglected in many cases because the content of the NG modes respects $S U(5)$ symmetry.) For the mass matrices of the other representation fields, $\mathbf{1 0}$ and $\mathbf{2 4}$ of $S U(5)$, we can roughly discuss the effect of $\epsilon \ll 1$. For the mass matrix of $\mathbf{1 0}$ and $\overline{\mathbf{1 0}}$ fields, the content of the NG modes respects the $S U(5)$. Therefore, the effect of $\epsilon \ll 1$ can be small in many cases, althogh the effect depends on the concrete models. (In the model in Table 8 , the effect can be neglected.) For the mass matrix of $\mathbf{2 4}$ fields, the NG modes are $(\mathbf{3}, \mathbf{2})_{-\frac{5}{6}}$ and $(\overline{\mathbf{3}}, \mathbf{2})_{\frac{5}{6}}$ under the SM gauge group. Therefore, these modes become heavier when $\epsilon \ll 1$. In summary, the effect of the deviation from the effective charge, $\epsilon$, on the gauge coupling unification appear in mass

spectrums of the doublet and $(\mathbf{3}, \mathbf{2})_{-\frac{5}{6}}$ pairs by enhancement factors, $\epsilon^{-1}$, in this model. The enhancement factor in the spectrum of the doublet reduces the disagreement of the gauge couplings caused by the large effective mass of the colored Higgs, $m_{\text {eff }} \gg \Lambda$.

\section{References}

[1] F. Wilczek and A. Zee, Phys. Lett. B70, 418 (1977), (Erratum-ibid.72B, 504 (1978)); S. Pakvasa and H. Sugawara, Phys. Lett. B73, 61 (1978) ; A. De Rujula, H. Georgi and S.L. Glashow, Annals Phys. 109, 258 (1977).

[2] L. Ibáñez and G.G. Ross, Phys. Lett. B332 (1994) 100; J.K. Elwood, N. Irges, P. Ramond Phys. Lett. B413, 322 (1997) ; N. Irges, S. Lavignac, and P. Ramond, Phys. Rev. D 58, 035003(1998); C.H. Albright and S. Nandi, Mod. Phys. Lett. A11, 737 
(1996); Phys. Rev. D 53, 2699 (1996); Q. Shafi and Z. Tavartkiladze, Phys. Lett. B459, 563 (1999); ibid B482, 145 (2000); ibid B487, 145 (2000); Nucl. Phys. B573, 40 (2000); Y. Nomura, T. Sugimoto, Phys. Rev. D 61, 093003 (2000); K.-I. Izawa, K. Kurosawa, Y.Nomura, T.Yanagida, Phys. Rev. D 60, 115016 (1999).

[3] M. Bando, T. Kugo, Prog. Theor. Phys. 101, 1313 (1999); M. Bando, T. Kugo, and K. Yoshioka, Prog. Theor. Phys. 104, 211 (2000).

[4] N. Maekawa, Prog. Theor. Phys. 106 (2001)401; arXiv:hep-ph/0110276.

[5] M. Bando and N. Maekawa, Prog. Theor. Phys. 106 (2001) 1255.

[6] N. Maekawa and T. Yamashita, Prog. Theor. Phys. 107, 1201 (2002).

[7] N. Maekawa and T. Yamashita, Prog. Theor. Phys. 110, 93 (2003).

[8] Z. Berezhiani, Phys. Lett. B150, 177 (1985); T. Blazek, S. Raby, and K. Tobe, Phys. Rev. D 62, 055001 (2000); R. Kitano and Y. Mimura, Phys. Rev. D 63, 016008 (2001); G.G. Ross and L. Velasco-Sevilla, Nucl. Phys. B653,3 (2003); S. Raby, Phys. Lett. B 561, 119 (2003); M.-C. Chen and K.T. Mahanthappa, Phys. Rev. D 68, 017301 (2003).

[9] F. Borzumati and A. Masiero, Phys. Rev. Lett. 57, 961 (1986); R. Barbieri, L.J. Hall, and A. Strumia, Nucl. Phys. B 445, 219 (1995); J. Hisano, T. Moroi, K. Tobe, M. Yamaguchi, and T. Yanagida, Phys. Lett. B 357, 579 (1995); J. Hisano, T. Moroi, K. Tobe, and M. Yamaguchi, Phys. Rev. D 53, 2442 (1996); J. Hisano and D. Nomura, Phys. Rev. D 59, 116005 (1999); J. Sato and K. Tobe, Phys. Rev. D 63, 116010 (2001); A. Masiero, S.K. Vempati, O. Vives, Nucl. Phys. B 649, 189 (2003).

[10] D.B. Kaplan and M. Schmaltz, Phys. Rev. D 49, 3741 (1994); L. J. Hall and H. Murayama, Phys. Rev. Lett. 75, 3985 (1995) R. Dermisek, S. Raby, Phys. Rev. D 62015007 (2000); K. Hamaguchi, M. Kakizaki and M. Yamaguchi, Phys. Rev. D68, 056007 (2003); Tatsuo Kobayashi, Jisuke Kubo and Haruhiko Terao, Phys. Lett. B568, 83 (2003).

[11] Y. Nir and N. Seiberg, Phys. Lett. B309, 337 (1993); Y. Nir and G. Raz, Phys. Rev. D 66, 035007 (2002).

[12] M. Dine, A. Kagan, and R. Leigh, Phys. Rev. D 48, 4269 (1993); A. Pomarol and D. Tommasini, Nucl. Phys. B466, 3 (1996); R. Barbieri, G. Dvali, and L.J. Hall, Phys. Lett. B377, 76 (1996); R. Barbieri and L.J. Hall, Nuovo Cim. A 110, 1 (1997); K.S. Babu and S.M. Barr, Phys. Lett. B387, 87 (1996); R. Barbieri, L.J. Hall, S. Raby, and A. Romanino, Nucl. Phys. B493, 3 (1997); Z. Berezhiani, Phys. Lett. B417, 287 (1998); G. Eyal, Phys. Lett. B441, 191 (1998); R. Barbieri, P. Creminelli, and A. Romanino, Nucl. Phys. B559, 17 (1999); S.F. King and G.G. Ross, Phys. Lett. B520, 243 (2001).

[13] The Super-Kamiokande Collaboration, Phys. Lett. B 436, 33 (1998); Phys. Rev. Lett. 81, 1562 (1998). 
[14] The Super-Kamiokande Collaboration, Phys. Rev. Lett. 86, 5656 (2001); Phys. Lett. B 539, 179 (2002); SNO Collaboration, Phys. Rev. Lett. 89, 011301 (2002); 89, 011302 (2002); KamLAND Collaboration, Phys. Rev. Lett. 90, 021802 (2003).

[15] N. Maekawa, Phys. Lett. B 561, 273 (2003); arXiv:hep-ph/0304076 hep-ph/0402224.

[16] N. Maekawa, Phys. Lett. B521 (2001) 42.

[17] N. Maekawa and Q. Shafi, Prog. Theor. Phys. 109, 279 (2003).

[18] N. Maekawa, Prog. Theor. Phys. 107, 597 (2002); N. Maekawa and T. Yamashita, Prog. Theor. Phys. 108, 719 (2002); Phys. Rev. Lett. 90 (2003) 121801.

[19] N. Maekawa and T. Yamashita, Phys. Lett. B 567, 330 (2003); Phys. Rev. D 68, 055001 (2003).

[20] E. Witten, Phys. Lett. B 149, 351 (1984); M. Dine, N. Seiberg, and E. Witten, Nucl. Phys. B 289, 589 (1987); J.J. Atick, L.J. Dixon, and A. Sen, Nucl. Phys. B 292, 109 (1987); M. Dine, I. Ichinose, and N. Seiberg, Nucl. Phys. B 293, 253 (1987).

[21] M. Green and J. Schwarz, Phys. Lett. B149 (1984),117.

[22] F. Gabbiani, E. Gabrielli, A. Masiero and L. Silvestrini Nucl. Phys. B477, 321 (1996).

[23] C.D. Froggatt and H.B. Nielsen, Nucl. Phys. B147, 277 (1979).

[24] S. Dimopoulos and F. Wilczek, NSF-ITP-82-07; M. Srednicki, Nucl. Phys. B202 (1982) 327.

[25] S.M. Barr and S. Raby, Phys. Rev. Lett. 79 (1997) 4748. 